\title{
Common and distinct patterns of terminal modifications to mirtrons and canonical microRNAs
}

\author{
JAKUB O. WESTHOLM, ${ }^{1}$ ERIK LADEWIG, ${ }^{1}$ KATSUTOMO OKAMURA, NICOLAS ROBINE, and ERIC C. LAI ${ }^{2}$ \\ Department of Developmental Biology, Sloan-Kettering Institute, New York, New York 10065, USA
}

\begin{abstract}
Nucleotide modifications to microRNAs or their precursors can influence their processing and/or activity. A challenge to their analysis is the lack of independent references for the termini generated by primary processing; typically, these are empirically assigned as the most abundant mapped reads. Mirtrons offer such an independent measure since these microRNA hairpins are defined by splicing. Consequently, mirtron-derived reads that deviate from splice sites reflect modification following primary processing. Analysis in Drosophila revealed multiple modification patterns, including select alterations of 5' termini, many $3^{\prime}$ resection events, and unexpectedly abundant $3^{\prime}$ untemplated monouridylation. Resections occur on mature AGO1-loaded species, whereas uridylation occurs on pre-miRNAs but is compatible with dicing and AGO1 loading. Strikingly, we found many mirtrons whose modified reads are more abundant than those produced by primary processing. In some cases, these abundant modified reads matched the genome owing to fortuitous uridines in downstream flanking exons, thus highlighting the value of an independent reference for the primary-processed sequence. We could further extend the principle of abundant and preferred uridylation of mirtrons, relative to canonical pre-miRNAs, to Caenorhabditis elegans, mouse, and human. Finally, we found that 3' resection occurs broadly across AGO1-loaded canonical miRNA and star species. Altogether, these findings substantially broaden the complexity of terminal modification pathways acting upon small regulatory RNAs.
\end{abstract}

Keywords: microRNA; mirtron; resection; uridylation

\section{INTRODUCTION}

The microRNA (miRNA) pathway generates diverse $\sim 21-$ 24 nucleotide (nt) RNAs, which collectively mediate essential developmental and physiological processes in most eukaryotes. In animals, the bulk of miRNAs mature via a canonical pathway in which primary hairpin-containing transcripts are first cleaved by the nuclear RNase III Drosha to generate $\sim 65-80$-nt pre-miRNA hairpins (Yang and Lai 2011). Following their export from the nucleus, they are cleaved by cytoplasm RNase III Dicer to yield $\sim 22-\mathrm{nt}$ miRNA/miRNA* species, of which one strand is preferentially incorporated into a mature Argonaute (Ago) complex and guides it to regulatory targets. A variety of functional miRNA:target geometries have been defined in animal cells, but by far the dominant mode is for targets to exhibit $\sim 7$-nt Watson-Crick complementarity to positions $2-8$ of the

\footnotetext{
${ }^{1}$ These authors contributed equally to this work.

${ }^{2}$ Corresponding author.

E-mail laie@mskcc.org.

Article published online ahead of print. Article and publication date are at http://www.rnajournal.org/cgi/doi/10.1261/rna.030627.111.
}

miRNA, referred to as the seed (Lai 2002; Lewis et al. 2003; Brennecke et al. 2005).

In addition to the canonical biogenesis pathway, a growing number of miRNAs have been recognized to traverse atypical pathways, including Drosha-independent and Dicer-independent strategies (Yang and Lai 2011). Mirtrons comprise the most numerous alternative miRNA substrates; these are short hairpin introns that are spliced and debranched to generate pre-miRNAs independently of Drosha (Okamura et al. 2007; Ruby et al. 2007a; Babiarz et al. 2008; Berezikov et al. 2010). Following their dicing and loading into Argonaute proteins, they appear functionally indistinguishable from miRNAs derived from canonical substrates. There also exist variant "tailed" mirtrons for which only one end of the pre-miRNA is generated directly by splicing. In the case of Drosophila $3^{\prime}$ tailed mirtrons, the tail is removed by the RNA exosome (Flynt et al. 2010); the relevant nuclease(s) that processes mammalian $5^{\prime}$ tailed mirtrons (Babiarz et al. 2008; Glazov et al. 2008) has not yet been elucidated.

Recently, there have been several reports of how nucleotide addition affects miRNA biogenesis, stability, and/or activity (Kim et al. 2010). This was initially described in 
plants, where $3^{\prime}$ methylation of miRNAs and siRNAs protects them from degradation by a mechanism involving $3^{\prime}$ uridylation ( $\mathrm{Li}$ et al. 2005; Yu et al. 2005). In animals, the best-studied case is that of Lin-28-mediated 3' uridylation of the let-7 family miRNAs. Uridylation catalyzed by TUT4/ PUP2 prevents dicing of pre-let-7 and leads to its destabilization (Heo et al. 2008; Rybak et al. 2008; Hagan et al. 2009; Heo et al. 2009; Lehrbach et al. 2009); Lin-28 was also suggested to repress nuclear processing of pri-let-7 (Newman et al. 2008; Viswanathan et al. 2008). In another example, miR-122 is adenylated by the GLD-2 poly-A polymerase, which positively regulates its stability (Katoh et al. 2009). Genomewide analysis of mammalian and Drosophila short RNAs indicates that both $3^{\prime}$ uridylation and adenylation are detected on broad cohorts of miRNAs, with evidence for specific substrate preferences of the modifying enzymes (Burroughs et al. 2010; Chiang et al. 2010; Berezikov et al. 2011). Finally, a target-driven mechanism for $3^{\prime}$ tailing and trimming mediates the destabilization of miRNAs that encounter highly complementary targets in Drosophila and mammalian cells (Ameres et al. 2010). Altogether, these studies suggest a rich diversity of mechanisms that modify miRNAs following definition of their initial sequences by primary processing.

Studies of miRNA modification are facilitated by the large collections of short RNA reads now publicly available, which commonly reveal a variety of variant reads referred to as "isomiRs" (Ruby et al. 2007b; Wu et al. 2007; Morin et al. 2008; Seitz et al. 2008; Chiang et al. 2010; Berezikov et al. 2011). However, a challenge to mining such data comes in defining the parental base sequence with confidence. The typical strategy is to define the base sequence as the most frequently cloned and mappable short RNA. One might then consider longer reads that share the base ("prefix") sequence, but do not match the genome unless terminal nucleotides are removed, as putatively bearing untemplated additions. However, it is not straightforward to distinguish whether terminal heterogeneity is due to alternative primary cleavage by RNase III enzymes or to directed post-processing nucleolytic reactions (Fig. 1A). Moreover, given the limited vocabulary of the genome, there is a $25 \%$ chance that an untemplated nucleotide will incidentally match the genome, potentially obscuring certain addition events. On a more practical level, as miRNA $3^{\prime}$ end heterogeneity may potentially stem from normal in vivo RNA catabolism or may reflect subpar sample preparation, relatively little effort has been made to study directed $3^{\prime}$ trimming of miRNAs.

To distinguish possible causes of small RNA modification, external references on the base sequence generated by primary processing would be informative. Mirtrons offer this possibility, since their pre-miRNA termini are defined by splicing (Fig. 1A). Examination of miRNAs derived from Drosophila mirtrons revealed frequent evidence for both terminal additions and deletions (Fig. 1B). We discerned cases in which $5^{\prime}$ variation was generated post-primary
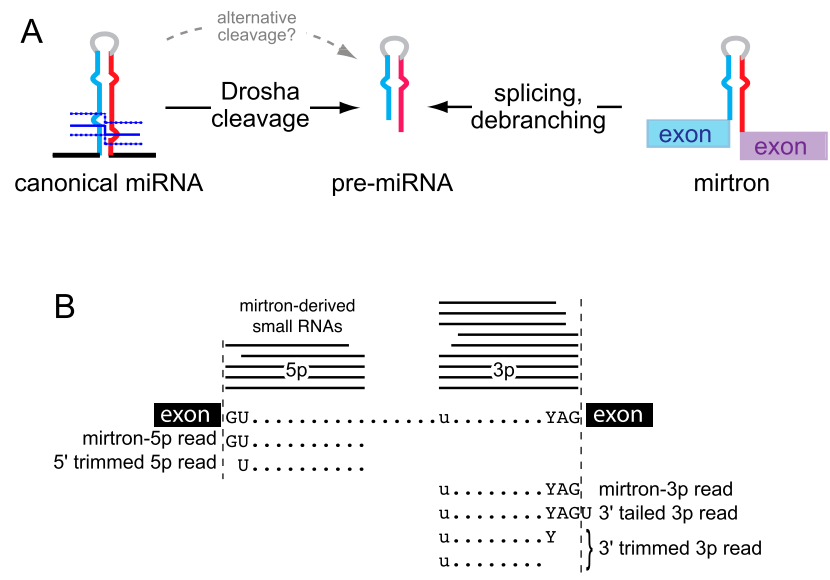

FIGURE 1. Rationale for studying modifications to mirtron-derived small RNAs. (A) The biogenesis of bulk miRNAs is initiated by cleavage of primary hairpin-containing transcripts into pre-miRNAs by the Drosha RNase III enzyme. Alternative registers of Drosha cleavage can produce variation in pre-miRNA termini, which are difficult to distinguish from post-cleavage modifications. In an alternate pathway, splicing bypasses Drosha cleavage to produce pre-miRNAs whose termini are thus strictly defined. (B) We exploit the precision of the initial step of mirtron processing to interpret the diversity of reads mapping to a given locus. Thus, $5 \mathrm{p}$ reads that do not begin with the "GU" splice donor can be inferred to have been trimmed, whereas $3 p$ reads that do not end in the "AG" splice acceptor can be inferred to have been modified, either by tailing or by trimming. Notably, one can assign nucleotide additions to the $3^{\prime}$ ends of mirtron- $3 p$ reads even in cases where the modification fortuitously matches the downstream exon sequence.

processing, as opposed to by alternative cleavage, suggesting a novel strategy to generate functional diversity. More unexpectedly, we observed mirtrons whose most abundant reads bore resected $3^{\prime}$ ends or carried $3^{\prime}$ untemplated additions. Motivated by these findings, we recognized a mechanism for preferred uridylation of mirtrons conserved across invertebrates and vertebrates and present evidence of trimming and tailing of canonical miRNAs, including untemplated reads that fortuitously match the genome. These data suggest that the spectrum of miRNA modifications is greater than is currently appreciated.

\section{RESULTS}

\section{$5^{\prime}$ isomiRs of mirtron $5 p$ and $3 p$ reads reveal distinct $5^{\prime}$ variation patterns}

Since the register for target recognition is set by the $5^{\prime}$ end of the miRNA, even single nucleotide shifts are expected to have substantial impact on the network of target transcripts captured by $5^{\prime}$ isomiRs relative to the dominant mature miRNA. Previous studies of $5^{\prime}$ isomiRs have assumed that the source of variation is alternative RNase III cleavage, by Drosha and/or by Dicer, but the possibility of $5^{\prime}$ decapitation could not be ruled out. Mirtrons offered a unique opportunity for analysis using an external reference. While 
the $5^{\prime}$ ends of their $3 \mathrm{p}$ reads are generated by Dicer and thus potentially amenable to alternative cleavage, the $5^{\prime}$ ends of $5 \mathrm{p}$ reads are generated by splicing and are, therefore, invariant in primary processing (Fig. 1).

By and large, the $5 p$ reads of Drosophila mirtrons are star species and accumulate to comparably low levels. However, the depth of available data permitted meaningful conclusions to be drawn (Berezikov et al. 2011), since we recovered hundreds to thousands of $5 p$ reads for most of the 31 annotated conventional mirtrons (Chung et al. 2011) in an aggregate set of 230 Drosophila small RNA libraries (Data set 1 ). The $5^{\prime}$ ends of mirtron-5p reads exhibited a very high rate of fidelity, since 28 of these exhibited $>90 \%$ precision of the initiating nucleotide, and 23 of those were defined with $>95 \%$ precision (Fig. $2 \mathrm{~A}, \mathrm{~B}$ ). This was true across the expression range contrasted with canonical miRNAs, which tend to be less precisely defined in the lower expression range.

However, exceptional mirtron-5p species had substantial numbers of reads beginning with the second intronic nucleotide ("xU") as opposed to the splice site ("GU").
Of these, miR-2500-5p was the most remarkable, since it generated many ( $>200$ reads), of which $69 \%$ had lost the initiating guanine nucleotide. This abundant " $\mathrm{xU}$ " $5^{\prime}$ isomiR of miR-2500-5p was preferentially associated with AGO1 effector complexes (17 "xU" reads and no "GU" reads in available AGO1 libraries), demonstrating it as a relevant functional species. miR-4909-5p was relatively lowly expressed but similarly exhibited a majority of " $\mathrm{xU}$ " reads; miR-1009-5p was relatively highly expressed and generated $\sim 20 \%$ " $x U$ " reads; both " $G U$ " and " $x U$ " isoforms were equally represented in AGO1-IP libraries. The fact of AGO1-loading for specific " $x U$ " reads contrasts with the general observation that AGO-loading purifies for specific $5^{\prime}$ ends (Seitz et al. 2008). Altogether, this provides evidence that specific small RNAs may acquire $5^{\prime}$ heterogeneity following their primary processing.

The $5^{\prime}$ ends of mirtron-3p species exhibited, on the whole, greater heterogeneity than their partner $5 p$ species (Fig. $2 \mathrm{C}, \mathrm{D}$ ), reflecting that Dicer-1 cleavage is less precise than splicing. We observed a general trend that less highly
31 Drosophila mirtrons

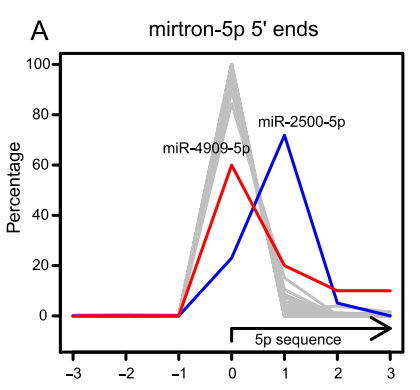

Position relative to canonical GU start of 5 'arm

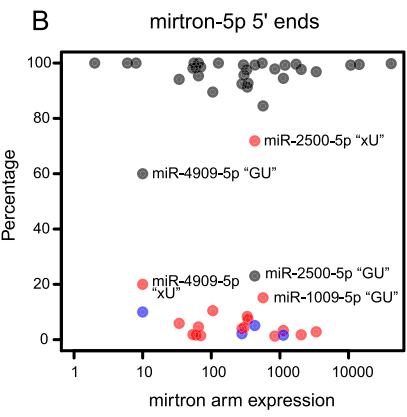

primary $5^{\prime}$ isomiRs

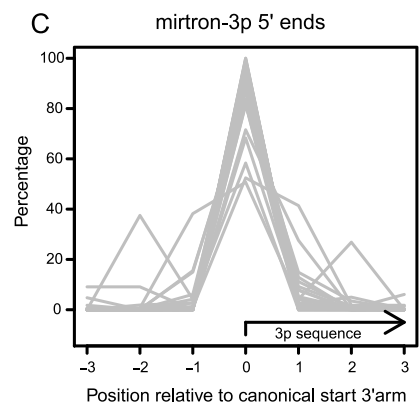

D mirtron-3p 5' ends

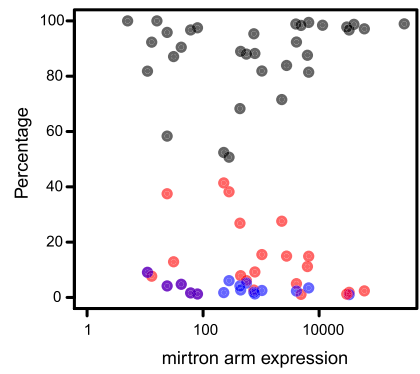

192 Drosophila canonical miRNAs
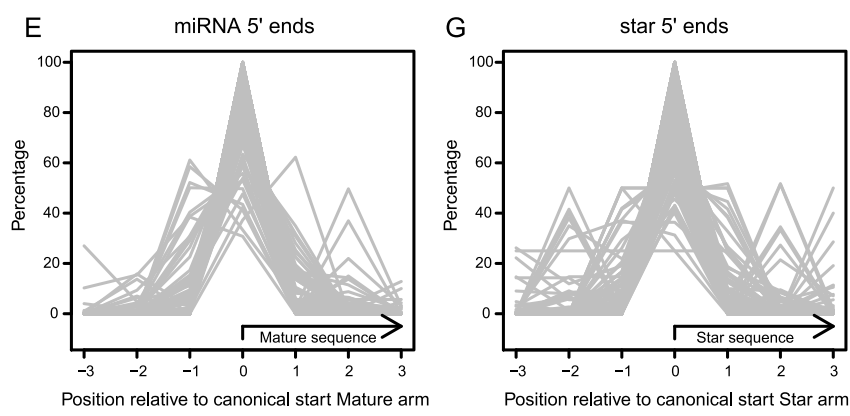

Position relative to canonical start Star arm

$\mathrm{F}$

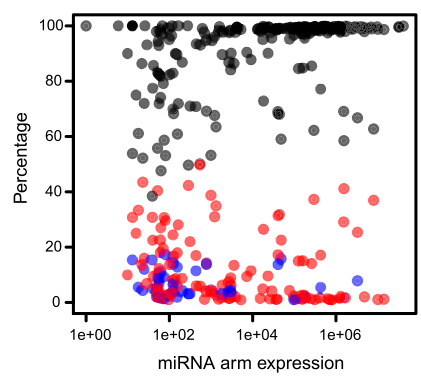

$\mathrm{H}$

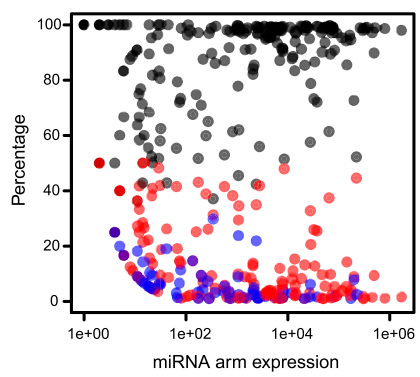

FIGURE 2. Comparison of the $5^{\prime}$ nucleotide precision among reads derived from Drosophila mirtrons and canonical miRNAs. Analyzed are reads from 31 mirtrons and 192 canonical miRNAs; all known loci were analyzed regardless of expression level. Note that the mature species from Drosophila mirtrons generally come from their $3 \mathrm{p}$ arms; therefore, the division of mirtron-5p/3p species is for the most part equivalent to mature/ star species of canonical miRNAs. (Top row) Relative distribution of $5^{\prime}$ starts of reads from individual loci; all reads of common $5^{\prime}$ end were grouped for analysis. (Bottom row) Frequency plots of $5^{\prime}$ isomiR distribution from individual loci. In black are the most abundant species (or the "GU" species from mirtron-5p reads), in red are secondary 5 ' isomiRs, and in blue are tertiary $5^{\prime}$ isomiRs; the latter two classes are shown when they contribute $>1 \%$ of the reads from a locus. $(A, B)$ Mirtron-5p reads generally exhibit high $5^{\prime}$ precision, even for very lowly expressed loci (i.e., few loci have secondary $5^{\prime}$ isomiRs $>5 \%$, and very few loci have tertiary $5^{\prime}$ isomiRs). However, reads from select loci have lost their $5^{\prime}$ nucleotides (" $x U^{\prime \prime}$ reads). (C,D) The 5 ' heterogeneity of mirtron-3p reads is greater than for mirtron-5p reads. ( $E, F$ ) The 5 ' heterogeneity of canonical mature miRNAs is greater than for mirtrons; this is evident on the lower end of expression. $(G, H)$ The $5^{\prime}$ ends of star species are the least well-defined among these classes of loci. 
expressed mirtron-3p species exhibited greater variation than more highly expressed species, consistent with a notion that less abundant miRNAs may have fewer targets and/or less pressure to adopt specific cleavage patterns. This fit with observations on the $5^{\prime}$ identities of species from canonical miRNA loci (Data set 2), for which mature miRNA $5^{\prime}$ ends are defined with greater overall precision than star species (Fig. 2E-H) but that certain highly expressed mature and star species exhibit atypically high variation (Ruby et al. 2007b; Berezikov et al. 2011). The observations from mirtrons imply that bulk $5^{\prime}$ variation of canonical miRNAs is likely a consequence of alternative processing by Drosha and/or Dicer-1 but that select species may acquire heterogeneity following their initial cleavages by RNase III factors via the action of a $5^{\prime}$-directed exoribonuclease.

\section{Trimming of 3' nucleotides from "long" mirtron duplexes}

The most frequently cloned miRNA/miRNA* species from canonical loci typically arrange as duplexes with $\sim 2$-nt $3^{\prime}$ overhangs, reflecting their biogenesis via Drosha/Dicer cleavage. In fact, hairpins whose small RNA duplexes lack 3' overhangs are often deemed potentially suspect as having derived from consecutive RNase III cleavages (Chiang et al. 2010; Berezikov et al. 2011). However, the precedent that some miRNA molecules utilize exonuclease trimming as a positive aspect of their biogenesis (Yang and Lai 2011) suggests that the lack of appropriate duplex ends is not necessarily inconsistent with genuine miRNA biogenesis.

We recently annotated the second intron of CG1718 as the mirtron mir-2535b (Chung et al. 2011), which was notable given that its third intron was earlier found to carry the mirtron mir-1007 (Ruby et al. 2007a). Curiously, the mir-2535b hairpin carries multiple large (5-nt) internal loops (Fig. 3A), a size of duplex imperfection that is rarely found even once in known canonical miRNAs. Despite this, mir-2535b generates a rather similar number of mature reads to mir-1007, including in AGO1-IP data sets, indicating that it generates genuine miRNAs. Since these mirtrons are processed from the same mRNA, this implied an unexpectedly similar dicing of pre-mir-1007 and premir-2535b.

As is typical for both mirtrons and canonical miRNAs, the $5^{\prime}$ ends of miR-2535b* (5p read) and mature miR$2535 \mathrm{~b}$ ( $3 \mathrm{p}$ read) are highly precise, while their $3^{\prime}$ ends are more heterogeneous. An abundant miR-2535b* species terminating on its 3 ' end in "...GUGG" forms a 2-nt 3' overhang with the precisely defined $5^{\prime}$ end of miR-2535b, while the $3^{\prime}$ end of miR-2535b extending to the splice acceptor "...GCAG" similarly forms a 2-nt 3' overhang. This defines these species as the primary Dicer-1 products, a notion supported by the recovery of 17 reads corresponding precisely to the phased terminal loop generated by mirtron cleavage. However, this duplex comprises species that are exceptionally large for Dicer products, with lengths of 25 and $26 \mathrm{nt}$ (Fig. 3A). We infer that the large internal loops of the mir-2535b hairpin are bulged out to present a compact form that is cleaved by Dicer-1.

We noticed a series of shorter reads representing $3^{\prime}$ terminal truncations of miR-2535b* and miR-2535b. A range of $3^{\prime}$ shortened reads are commonly observed in deeply sequenced data, but they are usually less abundant than the Dicer-cleaved species. In contrast, both products of the mir-2535b mirtron exhibited a continuous series of $3^{\prime}$ termini, ranging across $4 \mathrm{nt}$ on the $5 \mathrm{p}$ side and across $6 \mathrm{nt}$ on the $3 \mathrm{p}$ side. Inspection of head AGO1-IP data revealed that the dominant mature miR-2535b products in effector complexes had 4-5 nt removed from the $3^{\prime}$ terminus generated from primary processing, bringing them to 21-22 in length (Fig. 3A). Such trimming also occurs with its partner mirtron mir-1007, for which AGO1 complexes usually contained mature miR-1007 species with $2 \mathrm{nt}$ removed, from $23 \mathrm{nt}$ to $21 \mathrm{nt}$.

We sought to identify similar examples of $3^{\prime}$ trimmed miRNAs in mammals, in particular, cases of extensive resection. The locus mir-5129 ("SJmc-94") was recently annotated as a canonical miRNA in mouse on the basis of a modest number of reads (24 total for the locus) (Spierings et al. 2011). Inspection of its genomic region showed that it is actually a candidate $5^{\prime}$ tailed mirtron of the metastasis activator Zeb2. Since its hairpin bears a 6-nt bulge on its $3 \mathrm{p}$ arm, it might not be expected a priori that pre-mir-5129 can even serve as a Dicer substrate. Nevertheless, examination of several hundred mir-5129 reads collected from available public data sets (Data set 1 ) revealed that the $5^{\prime}$ ends of both $5 p$ and $3 p$ species were defined quite precisely, as is characteristic for confident miRNA loci (Fig. 3B). The third-most abundant read on the 5p arm extends to a 2-nt $3^{\prime}$ overhang with the precisely defined $5^{\prime}$ end on the $3 \mathrm{p}$ arm. The second- and first-most abundant $5 p$ species exhibited $3^{\prime}$ resection of 1-2 nt, but consequences on the $3 \mathrm{p}$ arm were more substantial. As a consequence of its stem imperfection, primary miR-5129-3p species are inferred to be $27 \mathrm{nt}$, a length unprecedented among miRNAs. However, $3^{\prime}$ resected species of 21,23 , and $22 \mathrm{nt}$ were all increasingly more abundant than the primary Dicer-cleaved species. Thus, maturation of this mammalian tailed mirtron involves resection of up to six $3^{\prime}$ nt, invoking a pathway analogous to "long arm" mirtrons in Drosophila.

Motivated by these observations, we searched for cases of extensive $3^{\prime}$ trimming of species from canonical miRNA loci. Here, the most compelling evidence is provided when an independent reference for RNase III cleavage positions is known. Insight can be gained from evidence of byproduct reads generated during miRNA biogenesis, namely $5^{\prime}$ and 3' miRNA offset reads (moRs) that mark Drosha cleavage (Shi et al. 2009) and terminal loop reads that mark Dicer cleavage (Ruby et al. 2007b). Although moRs and loop 

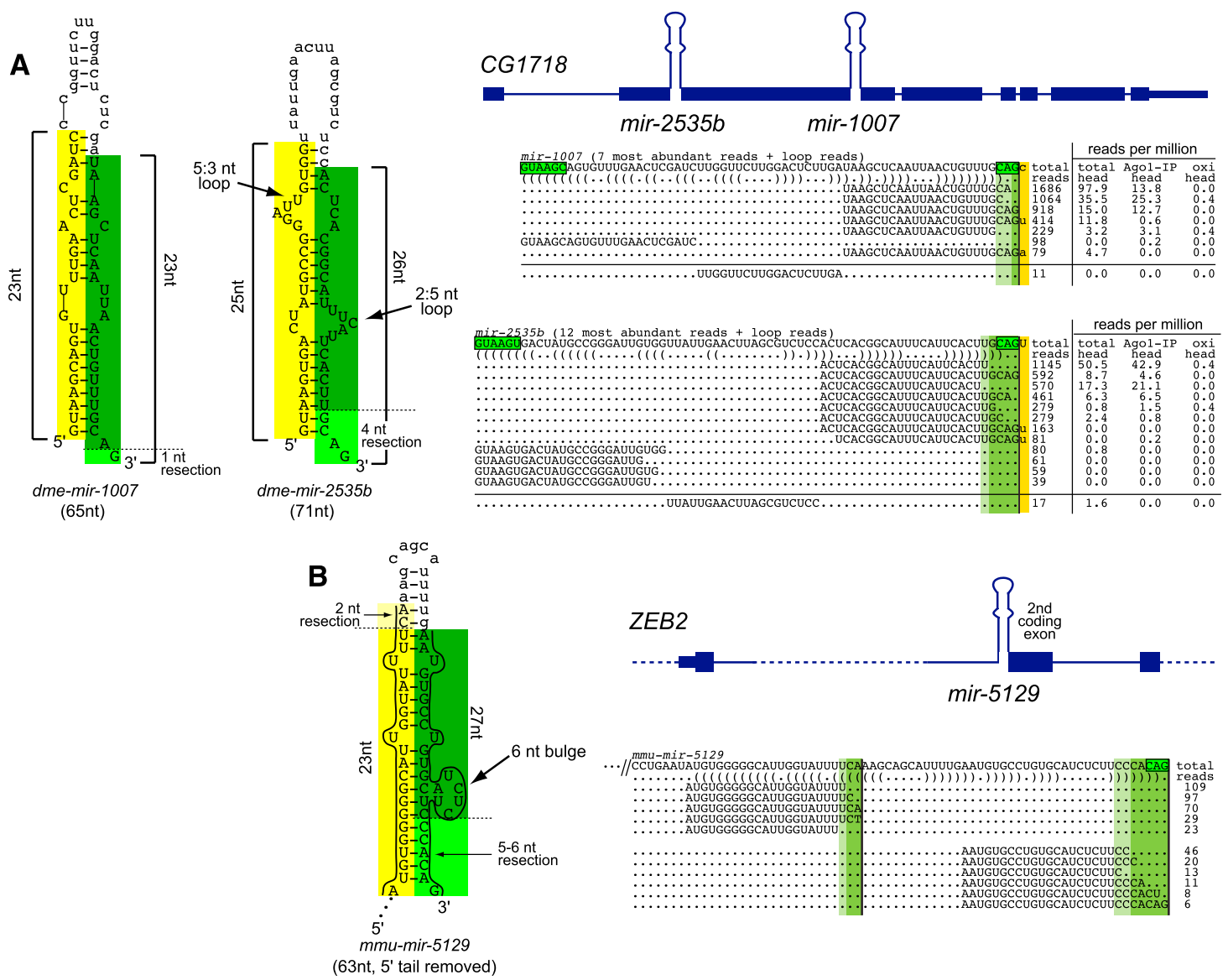

(63nt, 5 ' tail removed)

\section{C canonical miRNAs}
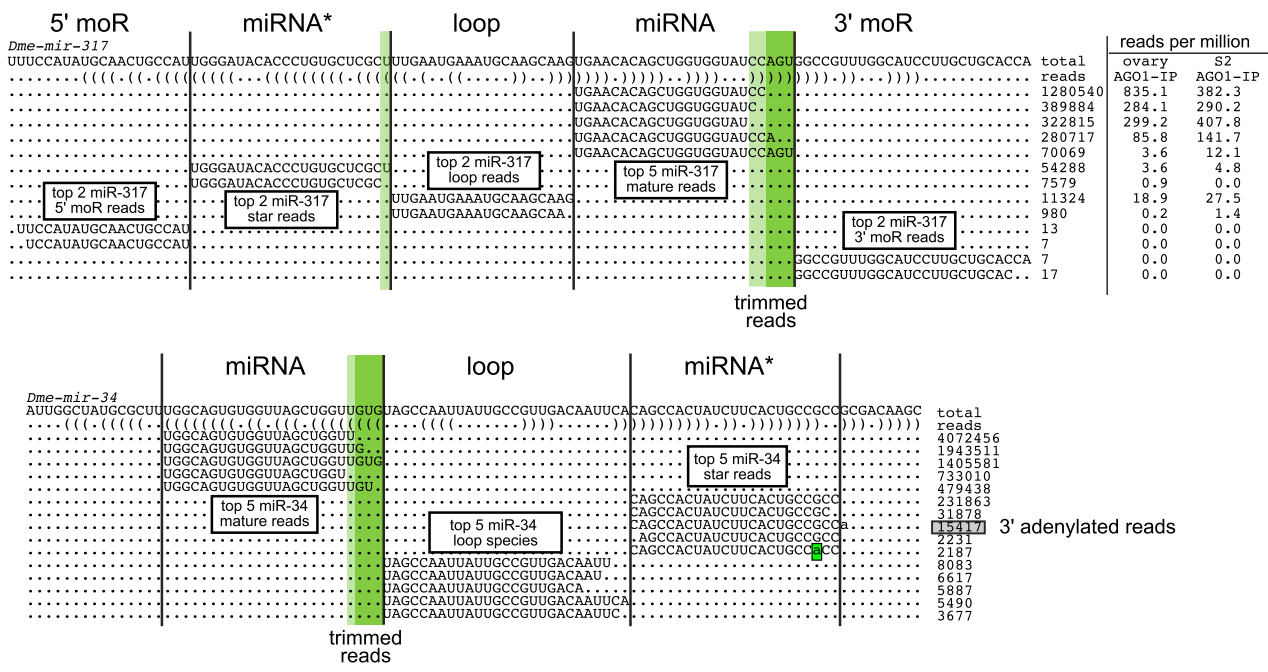

FIGURE 3. Extensive $3^{\prime}$ trimming of reads derived from mirtrons and canonical miRNA precursors with large stem imperfections. In the read alignments, $3^{\prime}$ resected nucleotides from termini initially defined by splicing or RNase III cleavage are highlighted in dark green for dominant species, and light green for minor species. (A) Drosophila CG1718 contains two mirtrons, mir-1007 and mir-2535b. These similarly sized introns fold into hairpins with typical 2-nt 3' overhangs, but the pre-mir-2535b hairpin exhibits large bulges. Nevertheless, the mature products of both mirtrons are loaded into AGO1 (GSM466489) and avoid AGO2 (oxidized libraries, GSM466488), as is characteristic of typical miRNAs. Dicer-1 cleavage of pre-mir$2535 b$ generates a "long" miRNA/star duplex with a 2-nt 3' overhang, with uridylated miR-2535b-3p species reaching 27 nt. However, the most abundant mature strand read in AGO1-IP is $22 \mathrm{nt}$ long, indicating $3^{\prime}$ resection of $4 \mathrm{nt}$ (and $5 \mathrm{nt}$ if they originated from the uridylated form). Note that miR-1007-3p is also resected in AGO1 complex. (B) Murine mir-5129 is a $5^{\prime}$ tailed mirtron located in the ZEB2 gene. This hairpin contains an unusually large 6-nt bulge, such that Dicer-cleavage generates a primary miR-5129-3p species of $27 \mathrm{nt}$; this is $3^{\prime}$ resected by $5-6 \mathrm{nt}$. (C) Detection of extensive $3^{\prime}$ resection of mature canonical miRNAs. Both the Drosha and Dicer-1 cleavage sites of Drosophila pri-mir-317 can be confidently assigned based on precise phasing of $5^{\prime}$ moR, miRNA*, loop, miRNA, and $3^{\prime}$ moR reads. Inspection of mature miR-317 reveals 4-5 nt resected from its $3^{\prime}$ end; these species are well-represented in AGO1-IP complexes (ovary AGO1IP, GSM609230 and S2 AGO1IP, GSM280088). With Dme-mir-34, the position of the Dicer-1 cleavage that defines the $3^{\prime}$ end of mature miR-34 can be confidently assessed based on the large number of terminal loop reads bearing a precise 5 ' end, which phases with the miR-34 species ending in "...UUGUG." The majority of miR-34 reads has been resected at their 3' ends by 3-4 nt. 
reads are generally quite rare, the massive amount of small RNA sequence data currently available in Drosophila (Berezikov et al. 2011) provides useful information.

For example, we observed a substantial discrepancy between the $3^{\prime}$ end of mature miR-317 (produced from its $3 \mathrm{p}$ arm) and its abundant $3^{\prime}$ moR reads (Fig. 3C). This indicated 3-5 nt were removed from the $3^{\prime}$ arm of primaryprocessed miR-317 in the dominant steady-state mature form, bringing it from $24 \mathrm{nt}$ down to 19-21 nt. Although it might be questioned whether such atypically short species are functional, even 19-20-nt miR-317 isomiRs were relatively abundant in AGO1-IP data from ovaries and S2 cells, indicating that they populate endogenous miRISC complexes. Otherwise, AGO1-IP complexes normally select for more conventional "miRNA-sized" species (Czech et al. 2008; Ghildiyal et al. 2010; Okamura et al. 2011).

Trimming also occurred with miRNAs generated from the $5 p$ hairpin arm, for which loop reads with stable $5^{\prime}$ ends provide independent evidence for the location of Dicer-1 cleavage. We previously noticed that mir-34 has a notable large population of cloned loops (Berezikov et al. 2011), which proves to exhibit a stable $5^{\prime}$ terminus (Fig. 3C). Comparison with mature miR-34 reads shows that $3 \mathrm{nt}$ are resected in the dominant species, and there is a substantial population with $4 \mathrm{nt}$ removed. Therefore, $3^{\prime}$ trimming can be detected for both mirtron and canonical miRNA loci.

\section{$3^{\prime}$ resection applies broadly to both mirtrons and canonical miRNAs in AGO1 complexes}

The above examples highlight dramatic cases in which multiple nucleotides are removed. We wished to know how broadly the phenomenon of $3^{\prime}$ resection applied. It has long been observed that the $3^{\prime}$ ends of miRNAs are relatively imprecisely defined, compared to their $5^{\prime}$ ends (Ruby et al. 2006; Landgraf et al. 2007; Ruby et al. 2007b; Morin et al. 2008). However, it has not been examined systematically whether such length variation is a property of mature Ago effector complexes. An alternative possibility is that such $3^{\prime}$ isomiRs may simply result from a preferred $3^{\prime}-5^{\prime}$ degradation pathway acting upon unloaded miRNAs. Since most previous analysis of $3^{\prime}$ heterogeneity utilized total RNA data sets (Ruby et al. 2007b; Morin et al. 2008; Fernandez-Valverde et al. 2010), these possibilities could not be distinguished. The recent availability of large amounts of Drosophila AGO1-IP data from our group (Okamura et al. 2011) and others (Czech et al. 2008; Ghildiyal et al. 2010) provided the opportunity to investigate this.

We first surveyed other Drosophila mirtrons for loss of terminal residues with respect to the splice acceptor sites (...AG), for which $3^{\prime}$ termini generated by primary processing could be assessed precisely (Fig. 1B). Since most Drosophila mirtrons generate mature species from their $3 \mathrm{p}$ arms, this analysis reports on overall $3^{\prime}$ variation of mature species from mirtrons. Of 28 loci with at least 10 mature $3 p$ reads, $12(43 \%)$ accumulated trimmed species that were collectively more abundant than full-length " $A G$ " terminating reads. When we restricted the analysis to AGO1-IP libraries, the overall abundance of trimmed reads was even higher, with $56 \%$ of the analyzed mirtron loci showing more trimmed reads, than reads that extend to the end of the intron (Fig. 4A). This provided evidence that these abundant trimmed reads are not simply degraded species but instead represent mature small RNAs in effector complexes.

We wished to compare the frequency of mirtron trimming with that of canonical miRNAs. This is not a trivial exercise owing to the heterogeneity of miRNA $3^{\prime}$ ends (Ruby et al. 2007b; Berezikov et al. 2011) and the fact that the most abundant read does not necessarily reflect the primary product of RNase III cleavage (e.g., Fig. 3). We, therefore, sought miRNA loci that exhibited highly invariant $5^{\prime}$ ends and possessed clear RNase III cleavage sites within well-duplexed regions, for which 2-nt $3^{\prime}$ overhangs could be judged, and culled loci for which moR reads suggested a possibility of alternate Drosha cleavage (see Materials and Methods). This yielded a set of 85 miRNA duplexes for which we could confidently define their primary processed forms (Data set 3 ).

Analysis of the frequency and extent of $3^{\prime}$ trimmed isoforms in AGO1 complexes, relative to their primary processed forms, revealed strikingly similar characteristics to mirtrons. About $40 \%$ of mature miRNAs exhibited a majority of reads in AGO1-IP data that were $3^{\prime}$ trimmed, and about $25 \%$ of star species were trimmed to a similar extent (Fig. 4B). Many loci generated trimmed reads in AGO1 complexes that were more abundant than the RNase III-defined species; such high extent of trimming was supported by a clear discrepancy with the $5^{\prime}$ ends of $3^{\prime} \mathrm{moR}$ reads (e.g., miR-11, miR-92b, miR-275, miR-190*, miR309, and miR-4; Data set 4). With lower cutoffs, one can observe that the strong majority of miRNA species exhibit at least some trimming in AGO1 complexes. We validated this phenomenon for the dramatic examples of miR-317 and miR-34. Unlike most other Drosophila miRNAs that exhibit 1 or 2 dominant mature species, these miRNAs exhibit 4-6 variant bands on Northern blots (Fig. 4C) that correlate with the heterogeneity observed in library analyses (Fig. 3C). Importantly, this wide distribution of mature miRNA lengths was observed in AGO1-IP Northern blots (Fig. 4C), confirming that it is a feature of functional effector complexes. By comparison, Northern analysis of bantam illustrated a miRNA that is only modestly trimmed, providing evidence that this process acts with some measure of specificity.

As with mirtrons, we infer that this resection occurs during the maturation of the miRISC, since the initial miRNA/ star duplexes generated exhibit typical 2-nt 3' overhangs that should be recognized by the AGO1 PAZ domain (Fig. $4 \mathrm{D})$. Moreover, the fact that both miRNA and star species are comparably trimmed indicates that this occurs post-dicing. 
A

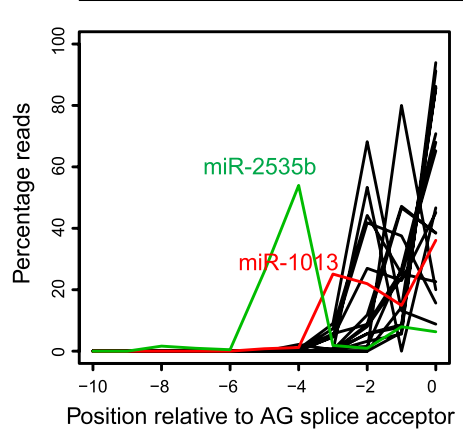

mirtrons in AGO1-IP

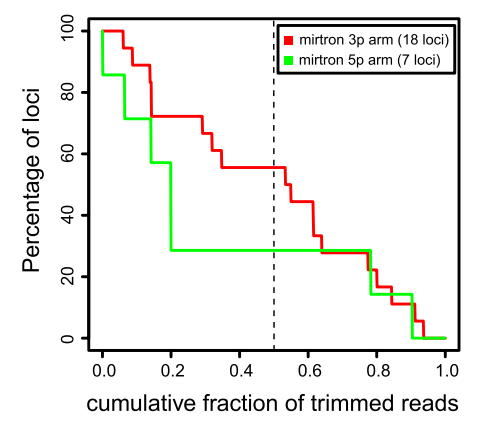

B

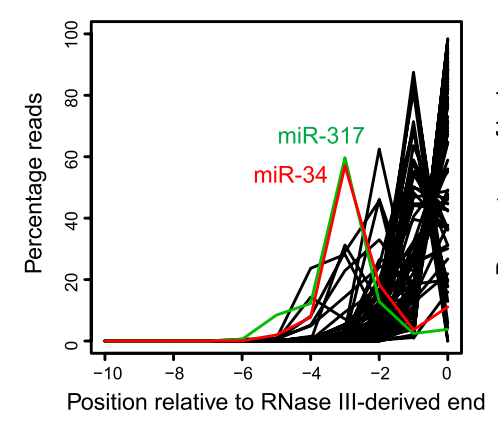

canonical miRNAs in AGO1-IP

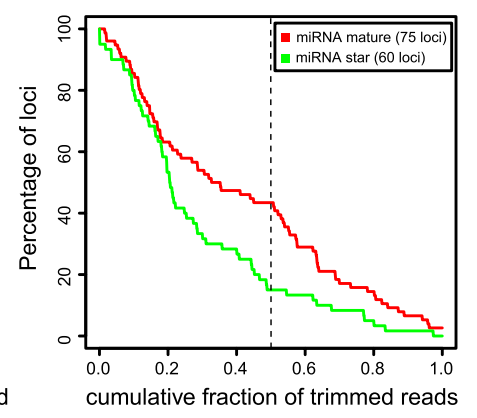

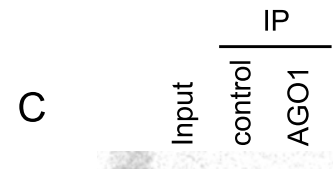

30
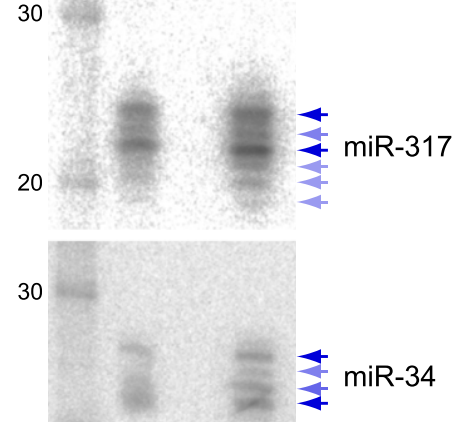

20

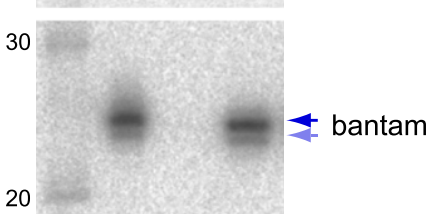

D

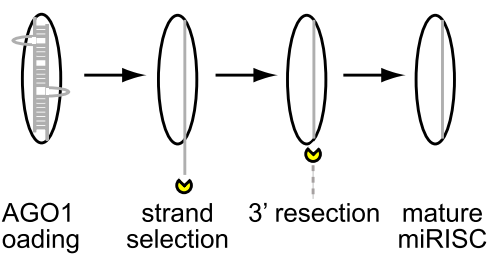

FIGURE 4. The $3^{\prime}$ resection acts broadly across mature AGO1-loaded species from D. melanogaster mirtrons and canonical miRNA loci. The primary-processed 3 ' ends of mirtron-3p species were defined by the "AG" splice acceptor. The termini of other categories of reads (mirtron-5p species and miRNA/star species from canonical loci) were judged from a vetted set of loci containing highly precise $5^{\prime}$ ends from which 2 -nt $3^{\prime}$ overhangs representing RNase III-cleaved products could be unambiguously defined. From these loci, reads from AGO1-IP data sets from ovaries, heads, and S2 cells (GSM609230, GSM466489, and GSM280088) were analyzed comprising the primary-processed species and 3' resected species; tailed reads were not considered in the tallies. Loci to which at least 10 full-length or trimmed reads mapped were included in the plot. $(A)$ The bulk of resection events to mirtron-3p species are in the 1-2-nt range, although select loci have incurred longer terminal deletions (e.g., miR$2535 \mathrm{~b}$ and miR-1013). Cumulative distribution shows that $3^{\prime}$ resection is a broad phenomenon among mirtron-derived miRNAs. $(B)$ The bulk of resection events to mature miRNAs are $1 \mathrm{nt}$, but a range of loci have incurred longer terminal deletions (e.g., miR-317 and miR-34). Cumulative distribution shows that the $3^{\prime}$ resection applies broadly to both mature miRNAs and star species, with overall rates that are comparable to mirtron-derived miRNAs. $(C)$ Northern confirmation of the existence of highly heterogeneous mature miR-317 and miR-34 species in AGO1 complexes. ( $D$ ) Model for $3^{\prime}$ resection posits that it occurs post-loading of the initial diced miRNA/star duplex. The $3^{\prime}$ resection is particularly evident on "long" species derived from hairpins with asymmetric imperfections (e.g., Fig. 3).

We conclude that the phenomenon of $3^{\prime}$ trimming applies quite broadly across functional AGO1-loaded miRNAs derived from both mirtrons and canonical miRNAs in Drosophila.

\section{Unexpectedly abundant $3^{\prime}$ uridylation of Drosophila mirtron-3p miRNAs}

Pre-miRNA hairpins and mature miRNAs can be uridylated and/or adenylated at their $3^{\prime}$ ends (Kim et al. 2010), and genomic studies indicate that both events are relatively common among miRNAs in mammals (Burroughs et al. 2010; Chiang et al. 2010) and Drosophila (Berezikov et al. 2011). Upon partitioning canonical miRNAs and mirtrons, we realized that a majority of the most highly modified miRNAs in Drosophila derived from mirtrons (e.g., miR-1003, miR-1008, miR-1010, and miR-1012). This was striking, given that mirtrons constitute only a small minority of well-expressed Drosophila miRNA genes that were included in our previous analysis (Berezikov et al. 2011).

We specifically analyzed mirtrons using an expanded set of 31 fly loci (Chung et al. 2011), by setting AG as the primary-processed $3^{\prime}$ terminus of mirtron- $3 p$ reads. Indeed, we observed extensive monouridylation in sequenced mirtron-3p reads, including ones for which the modified reads were the single most abundant species from the $3 \mathrm{p}$ arm of the mirtron (e.g., miR-1008 and miR-4919) (Fig. 5A; Supplemental Fig. S1, respectively). Notably, we identified 
several cases where the untemplated nucleotides actually matched genomic sequence. For example, a mirtron located in the intron of kuzbanian (mir2489) is flanked by four consecutive uridines in the downstream exon, whose presence in miR-2489-3p reads might otherwise be considered to be encoded genomically (Supplemental Fig. S1). In fact, there are far more monouridylated miR-2489 reads than reads defined by primary processing. Other cases of abundant, fortuitously matching, monouridylated mirtron-3p reads include miR-1014, miR-1016, miR-1005, and miR-1015 (Supplemental Fig. S3). Nevertheless, it was clear that these events were independent of the downstream exon, since uridylation was abundant regardless of the flanking exonic nucleotide. In total, 21/ 28 mirtrons exhibited $>25 \%$ uridylated 3 p reads with respect to "AG" reads, and $10 / 28$ had $>50 \%$ uridylated reads (Data set 5) (Fig. 5B).

When tabulated across all individual events of untemplated additions, uridylation and adenylation were clearly much more frequent than was addition of guanine or cytidine residues. However, uridylation was far more prevalent in terms of frequency of modified reads per locus and the number of affected loci. Consistent with previous results (Berezikov et al. 2011), we observed a bias for poly-U or poly-A additions, but $97.2 \%$ and $81.1 \%$ of tailed additions were single-nucleotide additions for mirtrons and miRNAs, respectively. Finally, monouridylation was strongly biased to mirtron- $3 p$ reads relative to mirtron-5p reads, implying the premiRNA hairpin as the substrate for modification (Supplemental Fig. S2A).

We were keen to exclude trivial explanations for the surprisingly high rate of mirtron 3' uridylation. For example, although we are not aware that this is the case, it seemed conceivable that some aspect of intron metabolism normally involves uridylation. We addressed this possibility by analyzing the distribution and frequency of tailed nucleotides among $3 \mathrm{p}$ reads from nonmirtron introns of 50-120 bp, collected from 230 Drosophila small RNA libraries (Data set 1).
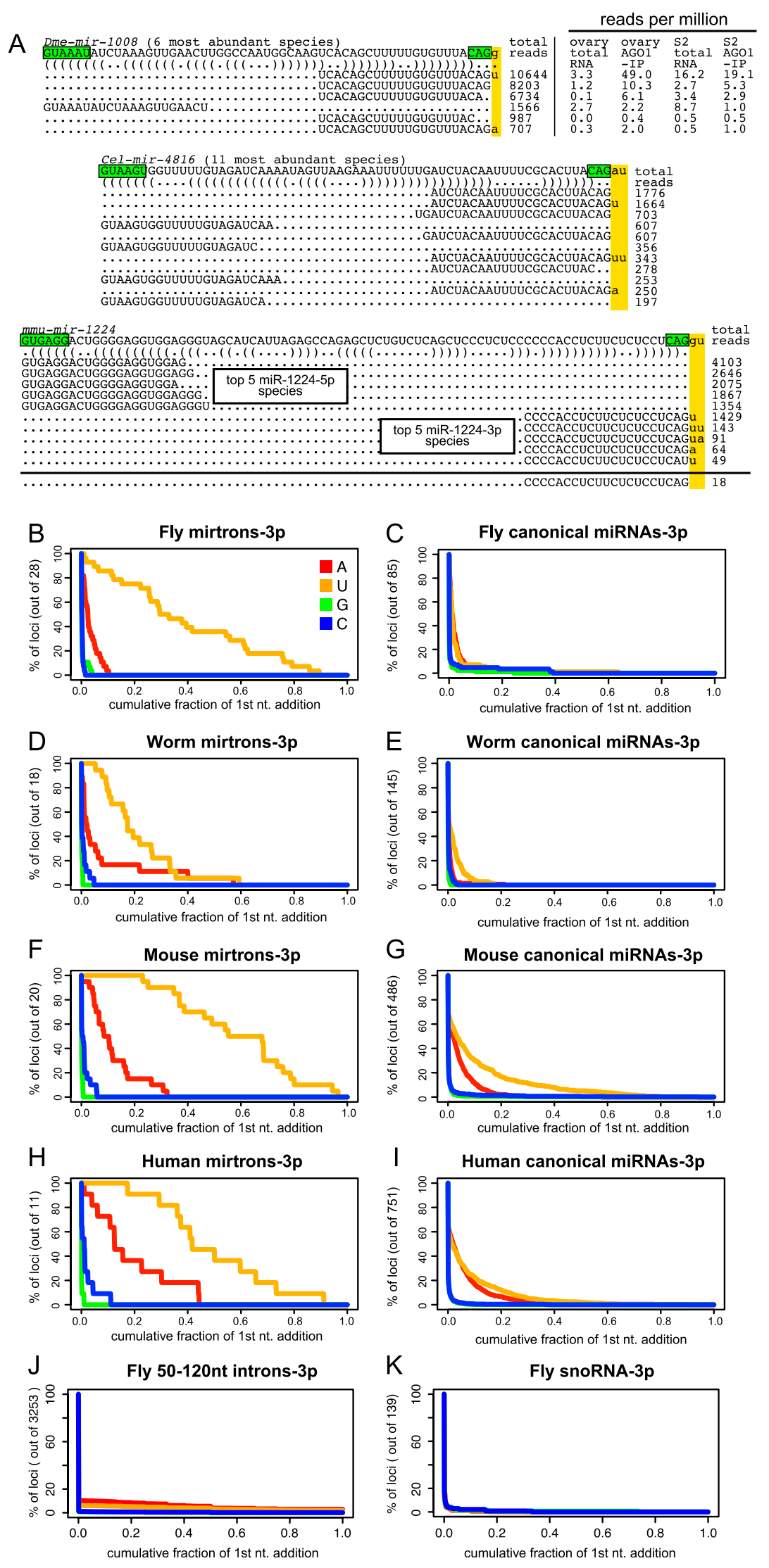

FIGURE 5. (Legend on next page) 
For this set, we considered all reads terminating in AG or extending 1-2 nt further, but we did not require an AG read to be present (since many mirtrons generate mostly modified $3 \mathrm{p}$ reads). We noted certain transcripts with retained or potentially misannotated introns, which yielded read patterns that spread evenly across putative intronexon boundaries. Since such reads are likely not untemplated additions and are expected to match the genome by virtue of transcription, we removed all such loci from analysis.

In total, we categorized 3253 short nonmirtron introns for which cloned $3 p$ reads were recovered. Of these, 2728 lacked nucleotide additions, indicating that cloned intron terminal fragments are not uridylated de facto or otherwise modified. Analysis of the tailing frequencies showed only a nominal overall bias of uridines and adenines over the other nucleotides (Fig. 5J). Therefore, the high rate of mirtron uridylation does not seem to stem from a general aspect of intron turnover.

We also examined additions to canonical miRNAs. To analyze Drosophila loci, we utilized the carefully vetted set of 3p species for which the Drosha cleavage sites had been examined and inferred not to be alternative (based on precision of the $5^{\prime}$ ends of corresponding $5 \mathrm{p}$ species and/or termini of moR reads; Data sets 2 and 3). This made it possible, as with the mirtron-3p analysis, to call untemplated nucleotides based on extension relative to the Drosha cleavage site, regardless of whether the nucleotide matched the genome or not. Canonical miRNAs preferentially exhibited $\mathrm{U}$ and $\mathrm{A}$ additions, as opposed to $\mathrm{G}$ and $\mathrm{C}$ additions, as observed earlier (Berezikov et al. 2011). However, the mild rate of $3^{\prime}$ uridylation (Fig. 5C) was nowhere near that of mirtron-3p reads. The distinction of mirtron and canonical miRNA uridylation was further emphasized in gene-by-gene plots (Fig. 6). We also performed a more conventional analysis using the larger set of 173 canonical Drosophila miRNAs, for which we used the standard practice of calling untemplated additions as nongenome matching reads that extend the most abundant $3 \mathrm{p}$ species. This yielded a similarly modest rate of terminal modification (Supplemental Fig. S2B).

Curiously, we did not observe a substantial difference in the modification rates of $5 p$ and $3 p$ canonical miRNA reads, even when dividing these into separate pools of mature $5 p$ vs. $3 p$ reads and star $5 p$ vs. $3 p$ reads to account for the differential sorting of Drosophila miRNA and star strands (Supplemental Fig. S2C). This suggests that there is no clear signal for preferential uridylation of canonical pre-miRNAs, as we concluded earlier (Berezikov et al. 2011). It appears that this signal in our previous analysis was driven by the inclusion of the highly modified mirtron class within the overall pool of miRNA species.

Finally, we examined a set of reads matching the $3^{\prime}$ ends of snoRNAs, which are an abundant class of short noncoding RNA that usually derive from spliced introns, although their termini are not directly defined by splicing, as with mirtrons. We mapped reads to 257 snoRNAs, and retained 222 loci to which short RNAs mapped specifically to the $3 p$ ends of the annotations (as opposed to extended heterogenously beyond the mapped snoRNA terminus). Of these, 139 snoRNA-3p reads bore at least some untemplated nucleotides. In total, we recorded 2,364,488 reads that matched the snoRNA terminus precisely, and only 18,894 with untemplated additions (Data set 6). These lacked detectable nucleotide preference (Fig. $5 \mathrm{~K}$ ), providing evidence that short intronic noncoding RNAs are not generically subject to uridylation or other terminal modification. Altogether, these results suggest that the uridylation machinery specifically recognizes mirtrons for efficient modification.
FIGURE 5. Conservation of frequent $3^{\prime}$ monouridylation of mirtron-3p species across animals. (A) Examples of highly uridylated mirtron-3p species from Drosophila, worm, and mouse. The 3' untemplated nucleotides, as judged with respect to "AG" splice acceptors, are highlighted by gold boxes. In the Drosophila example of mir-1008, quantification shows total reads collected from all libraries, as well as normalized read numbers from paired total RNA and AGO1-IP libraries from ovary (GSM280082, GSM609230) and S2 cells (GSM371638, GSM280088). These show that monouridylated mirtron-3p species are abundant in AGO1 complexes. In the C. elegans mir-4816 example, nearly half of the 5' isomiRs of miR-4816-3p that begin with "AUC..." have been uridylated; we also see substantial populations of di-uridylated reads, as well as mono-adenylated reads (the latter fortuitously match the genome). In the case of mmu-mir-1224, the five most abundant $5 p$ and $3 p$ species collected from all libraries are shown. Ninety-five percent of miR-1224-3p reads are uridylated; only 18 reads end precisely in the AG splice acceptor. $(B-K)$ Cumulative distribution function plots of single nucleotide additions following the $3^{\prime}$ ends of mirtron-3p reads, canonical miRNAs, bulk introns, and snoRNAs. $(B, C)$ Drosophila mirtrons and canonical miRNAs. $(D, E)$ Nematode mirtrons and canonical miRNAs. $(F, G)$ Mouse mirtrons and canonical miRNAs. $(H, I)$ Human mirtrons and canonical miRNAs. $(J)$ Bulk D. melanogaster short introns, which were carefully culled for instances of unspliced reads from annotated introns (Data set 2). (K) One hundred thirty-nine D. melanogaster snoRNAs to which precisely defined snoRNA-3p reads were recovered, with no more than 2-nt extensions beyond the annotated snoRNA terminus (Data sets 2 and 6).

\section{Conservation of preferred 3' uridylation of mirtrons across animals}

While uridylation of specific pre-miRNAs (e.g., pre-let-7) has been well-conserved across the Metazoa, the frequency and prevalence of Drosophila mirtron modification are far beyond most previous studied examples of miRNA modification in the steady-state pool. We were interested to assess whether this phenomenon was applicable to other species. We recently performed a metaanalysis of $>100$ small RNA libraries from Caenorhabditis elegans (Data set 1) from which the catalog of mirtrons was recently greatly expanded (Jan et al. 2010; Chung et al. 2011). The most abundantly 
A



B

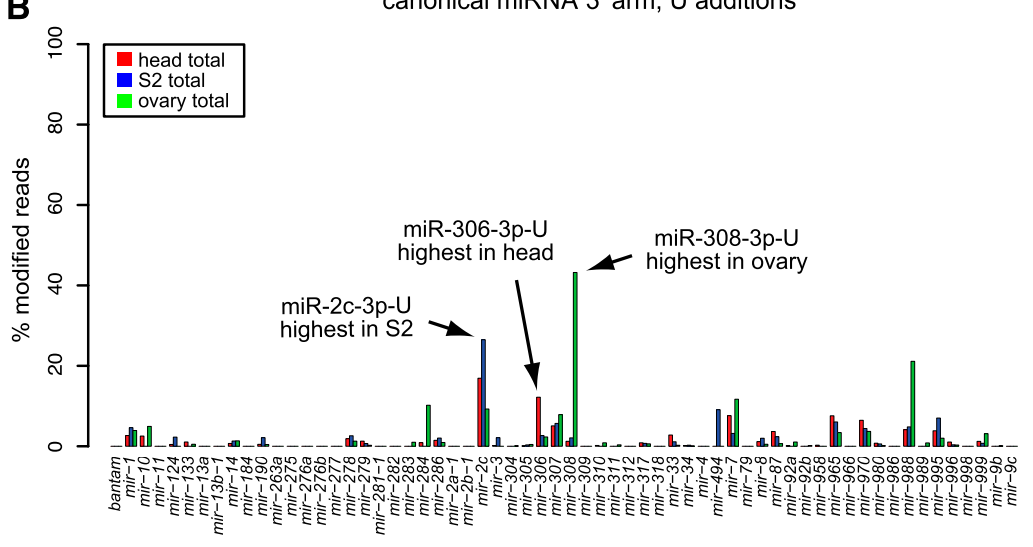

FIGURE 6. Tissue preference of mirtron uridylation in Drosophila. We analyzed total RNA libraries from heads (GSM609247, GSM609251, GSM286601, GSM286605, GSM322533, GSM322543), S2 cells (GSM272652, GSM361908, GSM371638), and ovaries (GSM609232, GSM628271, GSM609237, GSM628272, GSE24545, GSE24544). (A) The 3' uridylation of mirtron-3p reads was normalized relative to "AG" reads; trimmed reads were not considered in this analysis. The frequency of mirtron-3p uridylation was higher in ovaries compared to heads for all loci sampled (Wilcoxon $p$-value, $1.95 \times 10^{-3}$ ). (B) Analysis of canonical miRNA-3p species. We focused on 62 miRNA-3p's with at least 10 reads in each of the head/S2/ovary data sets. The graph highlights that, unlike mirtrons, few canonical miRNAs are uridylated above a few percent. Although a few miRNA-3p reads are relatively highly modified in ovaries, overall there is no comparable preference in this tissue. Wilcoxon $p$-values, head vs. $\mathrm{S} 2$ cells: 0.86 ; head vs. ovary: 0.91 ; ovary vs. S2 cells: 0.72 .

expressed mirtron in C. elegans is mir-62, to which we could map 225,497 full-length "AG" reads, but also 18,900 monouridylated reads (Supplemental Fig. S4). More substantially, in the case of mir-4816, there were 1776 "AG" reads and 1664 "AGU" reads of the dominant 5 ' isomiR (Fig. 5A). These observations motivated a systematic analysis of C. elegans canonical miRNAs and mirtrons. To do so, we analyzed the terminal modifications of an expanded set of C. elegans mirtrons, using a broad set of publicly available data that was recently used to annotate $145 \mathrm{ca}$ nonical miRNAs as well as 20 mirtrons (Jan et al. 2010; Chung et al. 2011). These studies showed that nematode mirtron-3p reads cumulatively exhibited a much greater proportion of $3^{\prime}$ uridylation than any other modification and that their rates of modification were much greater than for canonical miRNAs (Fig. 5D,E).

We also compared $3^{\prime}$ modification patterns in mammals. To do so, we collected 491 libraries of human small RNA data and 236 from mouse available from NCBI Gene Expression Omnibus or Short Read Archive, comprising 1.17 billion and 1.43 billion raw reads, respectively. The information on these mammalian data sets is summarized in Data set 1 . We analyzed 486 canonical mouse miRNAs and 751 canonical human miRNAs (http://www.mirbase.org). For mirtrons, we studied those human and mouse loci that were previously described explicitly (Berezikov et al. 2007; Babiarz et al. 2008; Chiang et al. 2010; Witten et al. 2010), including conventional and $5^{\prime}$ tailed mirtrons. All of the mouse loci showed clear mirtron-like patterns, but some human loci that we had reported (Berezikov et al. 2007) did not. Of these, mir-1225 and mir-1228 generated clear miRNA/star duplexes but appeared to have $3^{\prime}$ trimming of their $3 p$ reads, resulting in lack of "AG" or "AGU" reads. Potentially these loci receive $3^{\prime}$ trimming, as described in Drosophila (Flynt et al. 2010). Three loci generated small numbers of reads that did not provide confident assessment of dicing and were set aside (mir1231, mir-1234, and mir-1238). On the other hand, given our observation that at least one mouse locus annotated by Withoff and colleagues as a canonical miRNA (Spierings et al. 2011) was actually a mirtron (Fig. 3B), we examined their annotations in further detail. Indeed, in addition to mir-5129, the deep set of mouse small RNAs provided unambiguous evidence for five additional 5 tailed mirtron (mir-1966, mir-3572, mir-5107, mir-5132, and mir-5134), for which hairpins ending precisely in $3^{\prime}$ splice acceptor sites of well-annotated genes generated paired miRNA/star duplexes with appropriate 3 ' overhangs. Altogether, we retained a set of 20 mouse mirtrons and 11 human mirtrons that could be confidently assessed for tailing properties (i.e., had 10 or more $3 p$ reads).

Here, a difference between mammals and invertebrates was that mouse and human canonical miRNA reads exhibited higher overall rates of both uridylation and adenylation, consistent with previous studies (Burroughs et al. 2010; Chiang et al. 2010; Berezikov et al. 2011). The prevalence of adenylation were noticeably higher on mammalian mirtrons and canonical miRNAs (Fig. 5F-I), relative to flies and worms. Nevertheless, mirtrons in both mouse and human were subject to marked $3^{\prime}$ uridylation that was much more substantial than with canonical miRNAs (Fig. 5F-I; Supplemental Figs. S5, S6). Conserved mirtron mir-1224 serves to illustrate this point. The collected mouse small RNA data showed that $95 \%$ of some 
1800 miR-1224-3p species were uridylated; even the adenylated species for this locus outnumbered the unmodified "AG" reads (Fig. 5A). This trend was conserved in human, since we tabulated only six "AG" reads compared to 45 "AGU" reads for miR-1224-3p (Supplemental Fig. S6).

Overall, while only 38/486 (8\%) mouse and 25/751 (3\%) human canonical miRNAs exhibited $>40 \%$ uridylated reads, $14 / 20(70 \%)$ mouse mirtrons and 7/11 (41\%) human mirtrons exhibited $>40 \%$ uridylation in $3 p$ reads. In fact, when including adenylations into consideration, all 20 mouse mirtrons and all 11 human mirtrons exhibited $>40 \%$ terminal modifications of $3 p$ reads relative to "AG" reads (Data set 5). Notably, uridylated vertebrate mirtrons included ones for which the $5^{\prime}$ nucleotide of the downstream exon was uridine, and for which the uridylated forms were more abundant than unmodified reads ending in AG splice acceptors (Supplemental Figs. S5, S6). Such reads would not have been recognized as uridylated according to conventional mapping strategies. Altogether, these data demonstrate conservation of a pathway that preferentially uridylates the $3^{\prime}$ ends of mirtrons.

\section{Tissue-specific preference of mirtron uridylation}

Because miRNA modification factors such as Lin-28 exhibit temporal and spatial specificity, one may expect to see distinct patterns of miRNA modification across libraries. However, recent studies of uridylation of human miRNAs observed similar trends across many human cell lines (Burroughs et al. 2010). We took the opportunity to assess the ubiquity of mirtron modifications in Drosophila, using the abundant small RNA data from S2 cells, ovaries, and heads (Berezikov et al. 2011). This depth allowed sufficient reads from a broad selection of mirtrons to be analyzed. We focused on loci with at least 10 reads in all three locations and plotted the percentage of uridylated reads (relative to all reads where the genome matching portion ends at the AG splice acceptor) to facilitate comparison across tissues. Furthermore, we attempted to minimize sample preparation as an explanation for variation by analyzing S2 cell, ovary, and head small RNA data sets generated in our laboratory.

Although most mirtrons exhibited a high frequency of uridylation in all three settings (compared with canonical pre-miRNAs), we observed a clear tissue distinction in that ovaries exhibited significantly more mirtron uridylation, as did heads (Fig. 6A). This was individually true for all 10 mirtrons included in the analysis (paired Wilcoxon test p-value $1.95 \times 10^{-3}$ ), with six mirtrons having more than twice as much uridylation in ovaries compared to heads. The amount of uridylation in S2 cells was reproducibly intermediate to heads and ovaries. For example, miR-1008 and miR-1006 were strikingly $84 \%$ and $81 \%$ uridylated in ovaries, $66 \%$ and $52 \%$ in S2 cells, but only $28 \%$ and $5 \%$ in heads, respectively. Curiously, these trends appeared specific to mirtrons, since a similar analysis of canonical pre-
miRNAs did not reveal a comparable ovary-biased distribution of uridylated forms (Fig. 6B); the gene-by-gene analysis further emphasized the striking discrepancy in the frequency of uridylated mirtron-3p and canonical miRNA$3 \mathrm{p}$ reads. This distinction indicates that mirtrons and canonical miRNA hairpins are, as a rule, distinguished by the uridylation machinery, despite the fact that biochemical analysis suggested that debranched mirtrons and Droshacleaved pre-miRNAs appear functionally identical.

\section{Examples of genome-matching uridylation to canonical miRNA products}

One lesson learned from our mirtron studies was that there exist compelling cases of small RNAs bearing untemplated additions that fortuitously match the genome. Such cases would have escaped the notice of virtually all studies of miRNA modifications, since untemplated additions are usually analyzed de facto from the pool of nongenome matching reads. We were interested to see if this might mask some cases of miRNA uridylation.

As mentioned in the Introduction, $3^{\prime}$ uridylation of prelet-7 by lin-28/TUT4 is one of the best-studied examples of miRNA modification. In Drosophila, the second-most sequenced $5 \mathrm{p}$ (mature) species pairs with the most abundant 3p (star) species as a typical duplex with 2-nt $3^{\prime}$ overhangs, comprising precisely defined $5^{\prime}$ ends, representing the primary products of Drosha and Dicer-1 cleavage. The identity of Drosha cleavage sites was bolstered by the nearly one hundred $5^{\prime}$ moRs (comprising seven distinct $5^{\prime}$ ends but sharing the same $3^{\prime}$ end) and the $243^{\prime}$ moRs (spanning seven $3^{\prime}$ ends but sharing the same $5^{\prime}$ end). These $5^{\prime}$ and $3^{\prime}$ moRs phase precisely with both the $5^{\prime}$ end of the mature let- 7 and the inferred $3^{\prime}$ end of let- $7^{\star}(3 p)$ terminating in ...UUUCU. The availability of $5^{\prime}$ and $3^{\prime}$ moR evidence is informative, since the $3^{\prime}$ end of let-7-3p is followed by two additional genomically encoded uridines. We observed rare reads containing one or two "extra" uridines, which would normally be considered as "genomematching," but which in this case can be strongly inferred to represent uridylated forms of let-7-3p (Fig. 7A). Based on the $3^{\prime}$ uridylation of pre-let-7 in mammals and C. elegans, we infer this to be a signature of Lin-28-mediated modification.

The bantam locus is one of the most highly expressed miRNAs in Drosophila; unfortunately, the current lack of moR reads restricts an independent assessment of the Drosha cleavage sites. Nevertheless, the most abundant bantam $^{*}(5 p)$ and bantam (3p) reads pair with 2-nt $3^{\prime}$ overhangs at both duplex ends, and the only abundant $5^{\prime}$ isomiRs of bantam ${ }^{\star}$ are $1 \mathrm{nt}$ shorter. Therefore, we can assign mature bantam reads ending in three or more uridines as likely bearing untemplated additions, even though they match the genome (Fig. 7B). The numbers of reads inferred to bear genome-matching uridylation are highlighted in gold boxes. The inferred uridylation rate detected on mature 

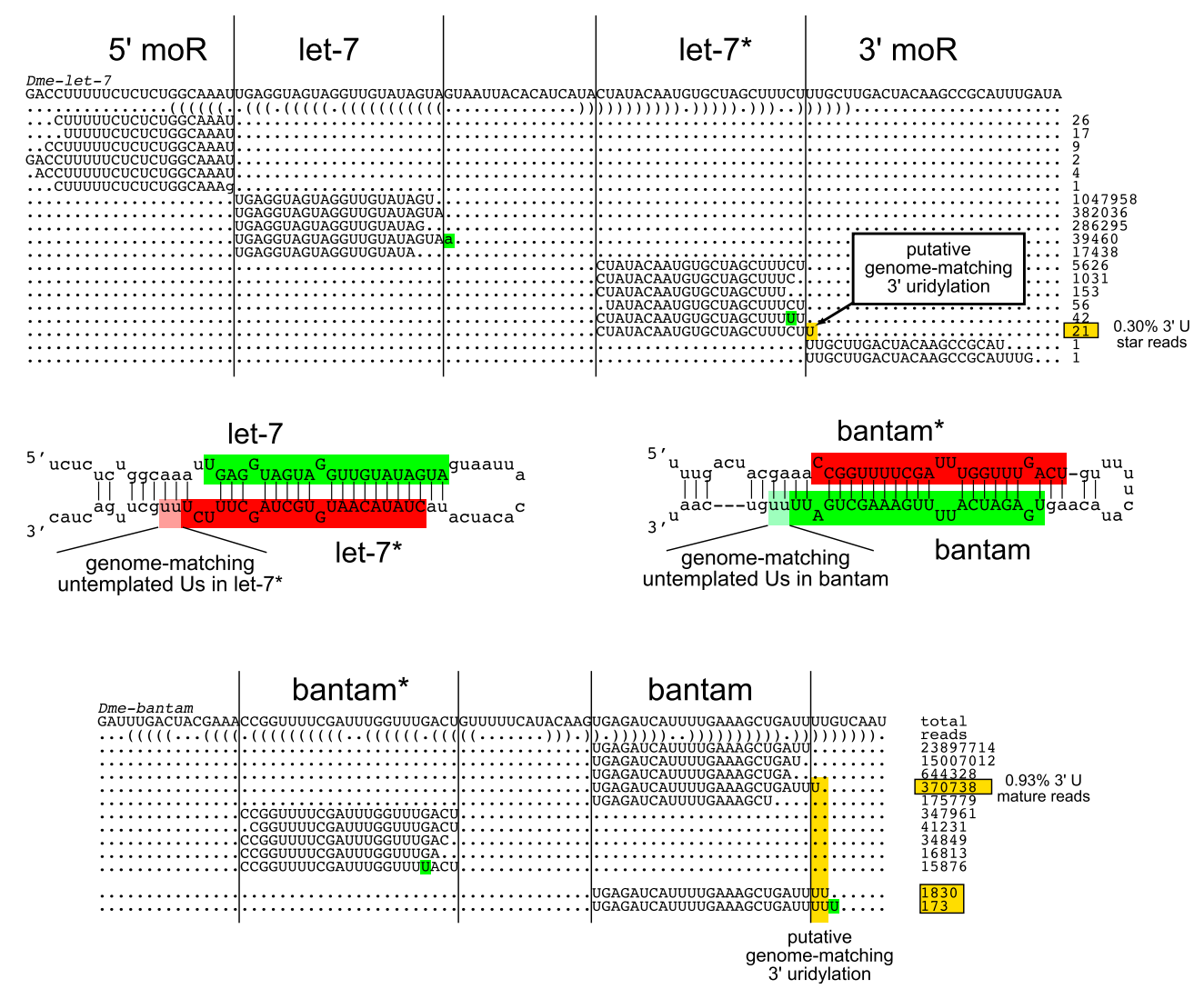

FIGURE 7. Examples of "hidden" uridylation of canonical miRNA products that fortuitously match the genome. Based on the read evidence presented, we infer two uridines to be downstream from both pre-let-7 and pre-bantam. (Top) The Drosha-cleavage sites of pri-let-7 can be confidently assessed based on the strict phasing of $5^{\prime}$ moR reads with mature let-7 (5p) reads and with the strict phasing of the most abundant let- $7^{\star}(3 p)$ species with $3^{\prime}$ moR reads. In addition, the $3^{\prime}$ end of the most abundant let- $7^{\star}$ species pairs with the precisely defined let-7 $5^{\prime}$ end with a 2 -nt $3^{\prime}$ overhang. Based on these observations, we can assign the sixth most abundant let- $7^{\star}$ species as likely bearing genome-matching, untemplated monouridylation. (Bottom) The Drosha-cleavage sites of pri-bantam cannot be independently assigned due to absence of moR reads; nevertheless, the most abundant bantam* $(5 p)$ and bantam (3p) reads pair with 2-nt $3^{\prime}$ overhangs at both duplex ends, and the only abundant $5^{\prime}$ isomiRs of bantam ${ }^{*}$ is $1 \mathrm{nt}$ shorter. Therefore, we can assign mature bantam reads ending in 3 or more uridines as likely bearing untemplated additions, even though they match the genome. The numbers of reads inferred to bear genome-matching uridylation are highlighted in gold boxes.

bantam is higher than that of let- $7^{\star}(0.93 \%$ vs. $0.30 \%$ reads, respectively). If uridylation of let- $7^{\star}$ can be taken as a proxy, then "genome-matching" modification of bantam may prove functionally significant.

\section{DISCUSSION}

\section{Implications for annotating nucleotide additions to miRNAs}

miRNAs bearing post-transcriptional nucleotide modifications are often considered to represent a small fraction of "base" reads, usually annotated as the most abundant genome-matching read generated by a given locus. By exploiting external references for base termini of small RNAs, i.e., splice donor and acceptor sites for mirtron-derived reads, we observed that modified miRNAs can be quite abundant. Indeed, nearly the entire steady-state output of several mirtron loci consisted of modified reads, tailed and/ or trimmed. Importantly, we identified loci for which abundant reads bearing untemplated additions masqueraded as "genome-matching" and others for which trimmed reads were the dominantly accumulating species. Indeed, the apparent small RNA evidence for many mammalian mirtrons may appear of borderline confidence when analyzing only genome-matching reads, but the picture changes dramatically when considering reads bearing untemplated monouridylation. These observations provide a rationale to reconsider mappings of canonical miRNAs in greater detail.

Lacking independent evidence for RNase III cleavage sites of canonical pri-miRNAs, other abundant modifications may be hidden among genome-matching reads. As the pool of Drosophila small RNA sequences has grown, an increasing number of miRNA offset (moR) and loop reads has been recovered (Berezikov et al. 2011). Although these are too rare to be informative for many loci, $5^{\prime} \mathrm{moR}$ and $3^{\prime}$ moR species often phase nicely with miRNA/miRNA* species, and intervening phased loops can also be observed 
when they happen to fall within the cloning range (Ruby et al. 2007b; Berezikov et al. 2011). Careful consideration of such references allowed us to determine that $3^{\prime}$ trimming of canonical miRNAs in AGO1 complexes is relatively common. We also present compelling examples of genome-matching uridylation events of star (let-7-3p) and mature (bantam$3 p)$ miRNA species, which are suggestive as functionally significant, at least given the known conservation of pre-let-7 uridylation between invertebrates and mammals. We speculate that availability of more comprehensive external references for Drosha and Dicer cleavage sites should broaden our appreciation of miRNA modification.

\section{Resection mechanisms for small RNA 3' end formation}

Intuitively, one might suspect that exonucleases are more likely to down-regulate rather than promote miRNA biogenesis. Indeed, both $3^{\prime}-5^{\prime}$ and $5^{\prime}-3^{\prime}$ exonucleases are known to antagonize miRNA stability (Kim et al. 2010). However, recent reports have uncovered positive roles for exonucleases in miRNA maturation. For example, in a variant pathway termed $3^{\prime}$ tailed mirtron biogenesis, splicing of mir-1017 generates a hairpin with a $3^{\prime}$-terminal extension that is removed by the nuclear RNA exosome to yield the pre-miRNA intermediate (Flynt et al. 2010). Reciprocally, mir-451 is a conserved vertebrate miRNA locus that is processed by Drosha to generate a short hairpin that is too short to be a Dicer substrate; instead, its 42-nt pre-miRNA hairpin is directly loaded into Slicer Ago2 (Cheloufi et al. 2010; Cifuentes et al. 2010; Yang et al. 2010). Ago2-mediated cleavage of pre-mir-451 yields a 30-nt RNA whose $3^{\prime}$ end is susceptible to a potentially cytoplasmic nuclease, thereby generating the dominant 23-nt product. In this study, we found that miRNAs derived from mirtrons and canonical miRNAs are broadly subject to $3^{\prime}$ trimming, likely within mature miRISC complexes. Such resection is often by 1 or 2 nt but is accentuated in certain mirtrons for which Dicer cleavage yields abnormally long species in the range of 24-27 nt (Fig. 4D). Therefore, in addition to well-known roles for RNase III endonucleases in the miRNA pathway, various nuclear and cytoplasmic exonucleases also play positive roles in the biogenesis of certain miRNAs.

Roles for ribonucleases other than RNase III enzymes are imperative for piRNAs, which are 24-30-nt RNAs bound by a germline-specific clade of Argonautes known as the Piwi proteins. Very little is known about their biogenesis, except that an amplification system known as "ping-pong" cleaves complementary targets (usually transposable elements) and thereby defines the $5^{\prime}$ ends of complementary piRNAs (Brennecke et al. 2007; Gunawardane et al. 2007). Otherwise, it is not known how piRNA 3' ends, or the 5' ends of nonamplified primary piRNAs, are defined except that that they do not seem to involve RNase III enzymes. Since the three different Piwi proteins in flies and in ver- tebrates carry distinct sizes of piRNAs (Siomi et al. 2011), it has been speculated that following definition of pre-piRNA $5^{\prime}$ ends (either by loading preference of $5^{\prime}$ uridine or as specified by ping-pong cleavage), the protruding $3^{\prime}$ ends might be reduced by a common nuclease. We imagine the same may apply to miRNA species whose size specified by Dicer cleavage is "too long" and are consequently trimmed to reflect the AGO1 footprint. The unexpected breadth of $3^{\prime}$ trimming is, in retrospect, consonant with the longstanding observation that miRNA $3^{\prime}$ ends are relatively imprecise in the steady-state pool (Ruby et al. 2006; Landgraf et al. 2007; Ruby et al. 2007b; Morin et al. 2008). The availability of large amounts of AGO1-IP data generated by our group (Okamura et al. 2011) and by others (Czech et al. 2008; Ghildiyal et al. 2010) confirms that $3^{\prime}$ resected miRNAs are frequent in mature miRISC effector complexes and thus make a major contribution to the diversity of miRNA sequences. It remains to be seen whether this is associated with functional variation.

\section{Implications of frequent mirtron modifications for their biogenesis}

Based on current knowledge, generation of the debranched mirtron yields a pre-miRNA mimic that is indistinguishable from bulk pre-miRNAs generated by Drosha cleavage, and their resultant mature products program functional AGO1 effector complexes as with conventional miRNAs. Nevertheless, our studies provide first evidence for molecular distinction in these substrates. Even though both types of hairpins contribute to the substrate pool for pre-miRNA uridylation, we observed that mirtrons are highly preferred substrates. Overall, we find that most mirtrons-including ones that are well-conserved and recently evolved, higherexpressed and lower-expressed-are strongly and preferentially uridylated relative to canonical pre-miRNAs in four species: Drosophila, C. elegans, mouse, and human.

The phylogenetic breadth of this phenomenon suggests that mirtron uridylation is of broad utility in metazoans. This modification does not promote mirtron degradation and is not inhibitory to mirtron dicing, as shown for prelet-7 (Heo et al. 2008, 2009). Furthermore, consistent with the recent discovery that metazoan Dicers measure predominantly from the $5^{\prime}$ phosphate (Park et al. 2011), as opposed to the $3^{\prime}$ hairpin terminus, we find that mirtron uridylation does not adjust Dicer cleavage since their $3 p$ products exhibit consistent size. The influence of distinctive mirtron uridylation on biogenesis or function merits investigation. Definitive evidence of this modification will come with identification of the relevant uridyltransferase and analysis of its knockdown or genetic deletion. As with the other features of mirtron modification described in this study, roles for nucleotide addition and removal to miRNAs and their precursors should have expanding consequences for small RNA biogenesis and function. 


\section{MATERIALS AND METHODS}

\section{Data sets, preprocessing, and annotations}

We collected reads from 75 Drosophila libraries generated in our laboratory, all of which are publicly available at the modENCODE Data Coordination Center (http://submit.modencode.org/submit/ public/list) or at NCBI Gene Expression Omnibus (GEO). We also collected other publicly available Drosophila melanogaster, C. elegans, mouse, and human small RNA data sets from GEO or the NCBI Short Read Archive. In total, these comprised 140 worm, 230 fly, 236 mouse, and 491 human libraries. The accession numbers of all data sets analyzed in this study are summarized in Data set 1 . MicroRNA annotations were downloaded from miRBase release 17, April 2011, along with instances of antisense miRNAs recently annotated in Berezikov et al. (2011).

Mirtrons were called based on annotations in fly (Chung et al. 2011), worm (Jan et al. 2010; Chung et al. 2011), mouse (Berezikov et al. 2007; Babiarz et al. 2008; Chiang et al. 2010; Witten et al. 2010), and human (Berezikov et al. 2007; Persson et al. 2011; Spierings et al. 2011). As discussed in the text, three human mirtrons earlier reported (Berezikov et al. 2007) failed to meet read evidence for confident annotation, whereas another cohort of six loci annotated as human canonical miRNAs proved to be $5^{\prime}$ tailed mirtrons (Spierings et al. 2011). Duplicated loci were removed from further analysis to avoid cross-matching, and Drosophila $3^{\prime}$ tailed mirtrons were excluded as they are not expected to generate $3 p$ "AG" reads (Flynt et al. 2010). Annotations of Drosophila introns and snoRNAs were obtained through the UCSC table browser (http://genome.ucsc.edu). For certain analyses, these loci were filtered further, e.g., on minimum numbers of mapping reads or lack of evidence of specificity in intron definition or snoRNA terminus. The loci included in each analysis are listed in Data set 2.

All of the raw sequence data were processed through a custom pipeline to facilitate preprocessing, filtering, and mapping to a reference genome. Briefly, reads were first trimmed of their $3^{\prime}$ sequencing adaptor with the fastx-clipper tool (http://hannonlab. cshl.edu/fastx_toolkit/). Those found with the adaptor were then filtered by size $\geq 17 \mathrm{nt}$, and mapped to their respective genome using Bowtie version 0.12.5, allowing for 0 mismatches (C. elegans WS190/ce6, D. melanogaster BDGP R5/dm3 minus U chromosome, Mus musculus NCBI37/mm9, and Homo sapiens GRCH37/ hg19). Unmapped reads were then iteratively trimmed up to $4 \mathrm{nt}$ from the $3^{\prime}$ end while attempting to map to the genome allowing for 0 mismatches. Information on all library processing is provided in Data set 1. All reads were then collected by locus, separated by library identity and presented in an html interface for further analysis. Individual gene pages for each species are available at http:// ericlailab.com/tail_and_trim/. For quantification, genomic mapping reads were normalized by the number of genomic locations to which they mapped. RNAshapes and RNAfold were used to predict RNA secondary structure.

\section{Terminal heterogeneity analysis}

To determine the heterogeneity of mirtron and miRNA reads (Fig. 2 ), we collected sense reads from both ends of the loci. All reads which occur within $4 \mathrm{nt}$ of the most abundant read were grouped and summed according to position. Mirtron reads were gathered according to the GT..AG splice donors and acceptors, while mature and star miRNA sequence locations were determined for each miRNA according to abundance of the reads.

To assess trimming of canonical miRNAs, we used a manually annotated set of miRNA duplexes (Data set 3). The annotation was done by first identifying duplexes where both $5 p$ ends were invariant ( $>90 \%$ of reads had the same start), and assuming a 2-nt $3 p$ overhang on the opposite arms. Loci where the read patterns supported overhangs of $2 \mathrm{nt}$ (or less, in the case of extensive trimming) were kept. Loci with less than 10 reads of the most common species, nonuniquely mapping reads, conflicting moR reads, or where the most abundant reads resulted in overhangs of $>2 \mathrm{nt}$ were removed. For mirtrons, the AG splice acceptor was used as the end of the full-length sequence.

Tailing of fly mirtrons and canonical miRNAs was analyzed using the same annotations as for trimming. To quantify tailing events, we counted reads that extended exactly one nucleotide beyond the annotated sequence ("monoadditions") relative to the number of read ending exactly at the end of the annotated sequence. Worm, human, and mouse mirtrons were annotated the same way as for fly, by setting the AG as the end of the canonical sequence. Worm, human, and mouse canonical miRNAs were annotated by letting the most abundant reads define the canonical sequences. Only loci with at least 10 reads ending at the defined $3^{\prime}$ site and a corresponding genome matching read were included in the analysis.

A control set of fly introns of length 50-120 nt was taken from the UCSC table browser and filtered to ensure that each AG acceptor site was represented only once, that each intron had at least one read mapping around the splice acceptor, and that no published (Chung et al. 2011) mirtrons were included. Introns with extensive read coverage indicating read-through were identified using the following proxy: If an intron had more reads that extend at least $4 \mathrm{nt}$ into the downstream exon than reads that matched the genome exactly up to the AG splice acceptor, it was removed from subsequent analysis. This removed 232 introns out of an initial pool of 3485 introns. Another control set of fly snoRNA sequences was obtained from the UCSC table browser. Loci with excessive read-through, defined as having reads that map across the $3^{\prime}$ boundary in excess of $10 \%$ of all $3^{\prime}$ reads, were removed from subsequent analysis. (See Data set 2 for details on intron and snoRNA loci.)

\section{Northern analysis}

Adult Drosophila heads were collected and homogenized in HepesNP-40 buffer (30 mM HEPES-KOH (pH 7.3), $150 \mathrm{mM} \mathrm{KOAc,}$ $2 \mathrm{mM} \mathrm{MgOAc}, 5 \mathrm{mM}$ DTT, $0.1 \%$ NP40, $1 \times$ Complete EDTA free) and cleared by a centrifugation at $20,000 \mathrm{~g}$ for $10 \mathrm{~min}$ at $4^{\circ} \mathrm{C}$. Protein $\mathrm{G}$ Dynabeads bound with $5 \mu \mathrm{g}$ normal rabbit IgG or antiAGO1 (Abcam) were incubated with the head extract for $90 \mathrm{~min}$ at $4^{\circ} \mathrm{C}$ and washed five times with Hepes-NP40 buffer. Coprecipitated RNA was extracted by Phenol/Chloroform/Isoamyl alcohol followed by ethanol precipitation and analyzed by Northern blotting. LNA (miR-34 and miR-317; Exiqon) and DNA (bantam; IDT) oligonucleotides were 5'-labeled and hybridized as previously described (Okamura et al. 2007).

\section{SUPPLEMENTAL MATERIAL}

Supplemental material is available for this article. 


\section{ACKNOWLEDGMENTS}

We thank the many researchers who deposited their small RNA data in public databases that made this meta-analysis possible. We thank Harrison Tsai for discussions during the course of this work. J.O.W. was supported by a fellowship from the Swedish Research Council. Work in E.C.L.'s group was supported by the Burroughs Wellcome Fund, the Alfred Bressler Scholars Fund, the Starr Cancer Consortium (I3-A139), and the NIH (R01-GM083300 and U01-HG004261).

\section{NOTE ADDED IN PROOF}

Following acceptance of this work, the Bonini and Zamore labs reported that the $3^{\prime}-5^{\prime}$ exoribonuclease Nibbler trims the $3^{\prime}$ ends of Drosophila miRNAs (Han et al. 2011; Liu et al. 2011).

Received September 28, 2011; accepted November 9, 2011.

\section{REFERENCES}

Ameres SL, Horwich MD, Hung JH, Xu J, Ghildiyal M, Weng Z, Zamore PD. 2010. Target RNA-directed trimming and tailing of small silencing RNAs. Science 328: 1534-1539.

Babiarz JE, Ruby JG, Wang Y, Bartel DP, Blelloch R. 2008. Mouse ES cells express endogenous shRNAs, siRNAs, and other Microprocessor-independent, Dicer-dependent small RNAs. Genes Dev 22: 2773-2785.

Berezikov E, Chung W-J, Willis J, Cuppen E, Lai EC. 2007. Mammalian mirtron genes. Mol Cell 28: 328-336.

Berezikov E, Liu N, Flynt AS, Hodges E, Rooks M, Hannon GJ, Lai EC. 2010. Evolutionary flux of canonical microRNAs and mirtrons in Drosophila. Nat Genet 42: 6-9.

Berezikov E, Robine N, Samsonova A, Westholm JO, Naqvi A, Hung JH, Okamura K, Dai Q, Bortolamiol-Becet D, Martin R, et al. 2011. Deep annotation of Drosophila melanogaster microRNAs yields insights into their processing, modification, and emergence. Genome Res 21: 203-215.

Brennecke J, Stark A, Russell RB, Cohen SM. 2005. Principles of microRNA-target recognition. PLoS Biol 3: e85. doi: 10.1371/ journal.pbio.0030085.

Brennecke J, Aravin AA, Stark A, Dus M, Kellis M, Sachidanandam R, Hannon GJ. 2007. Discrete small RNA-generating loci as master regulators of transposon activity in Drosophila. Cell 128: 10891103.

Burroughs AM, Ando Y, de Hoon MJ, Tomaru Y, Nishibu T, Ukekawa R, Funakoshi T, Kurokawa T, Suzuki H, Hayashizaki Y, et al. 2010. A comprehensive survey of $3^{\prime}$ animal miRNA modification events and a possible role for $3^{\prime}$ adenylation in modulating miRNA targeting effectiveness. Genome Res 20: 1398-1410.

Cheloufi S, Dos Santos CO, Chong MM, Hannon GJ. 2010. A dicerindependent miRNA biogenesis pathway that requires Ago catalysis. Nature 465: 584-589.

Chiang HR, Schoenfeld LW, Ruby JG, Auyeung VC, Spies N, Baek D, Johnston WK, Russ C, Luo S, Babiarz JE, et al. 2010. Mammalian microRNAs: Experimental evaluation of novel and previously annotated genes. Genes Dev 24: 992-1009.

Chung WJ, Agius P, Westholm JO, Chen M, Okamura K, Robine N, Leslie CS, Lai EC. 2011. Computational and experimental identification of mirtrons in Drosophila melanogaster and Caenorhabditis elegans. Genome Res 21: 286-300.

Cifuentes D, Xue H, Taylor DW, Patnode H, Mishima Y, Cheloufi S, Ma E, Mane S, Hannon GJ, Lawson N, et al. 2010. A novel miRNA processing pathway independent of Dicer requires Argonaute2 catalytic activity. Science 328: 1694-1698.
Czech B, Malone CD, Zhou R, Stark A, Schlingeheyde C, Dus M, Perrimon N, Kellis M, Wohlschlegel J, Sachidanandam R, et al. 2008. An endogenous siRNA pathway in Drosophila. Nature 453: 798-802.

Fernandez-Valverde SL, Taft RJ, Mattick JS. 2010. Dynamic isomiR regulation in Drosophila development. RNA 16: 1881-1888.

Flynt AS, Chung WJ, Greimann JC, Lima CD, Lai EC. 2010. microRNA biogenesis via splicing and exosome-mediated trimming in Drosophila. Mol Cell 38: 900-907.

Ghildiyal M, Xu J, Seitz H, Weng Z, Zamore PD. 2010. Sorting of Drosophila small silencing RNAs partitions microRNA* strands into the RNA interference pathway. RNA 16: 43-56.

Glazov EA, Cottee PA, Barris WC, Moore RJ, Dalrymple BP, Tizard ML. 2008. A microRNA catalog of the developing chicken embryo identified by a deep sequencing approach. Genome Res 18: 957-964.

Gunawardane LS, Saito K, Nishida KM, Miyoshi K, Kawamura Y, Nagami T, Siomi H, Siomi MC. 2007. A Slicer-mediated mechanism for repeat-associated siRNA 5' end formation in Drosophila. Science 315: 1587-1590.

Hagan JP, Piskounova E, Gregory RI. 2009. Lin28 recruits the TUTase Zcchc11 to inhibit let-7 maturation in mouse embryonic stem cells. Nat Struct Mol Biol 16: 1021-1025.

Han BW, Hung J-H, Weng Z, Zamore PD, Ameres SL. 2011. The 3'to- $5^{\prime}$ exoribonuclease Nibbler shapes the $3^{\prime}$ ends of microRNAs bound to Drosophila Argonaute1. Curr Biol 21: 1878-1887.

Heo I, Joo C, Cho J, Ha M, Han J, Kim VN. 2008. Lin28 mediates the terminal uridylation of let-7 precursor microRNA. Mol Cell 32: 276-284.

Heo I, Joo C, Kim YK, Ha M, Yoon MJ, Cho J, Yeom KH, Han J, Kim VN. 2009. TUT4 in concert with Lin28 suppresses microRNA biogenesis through pre-microRNA uridylation. Cell 138: 696-708.

Jan CH, Friedman RC, Ruby JG, Bartel DP. 2010. Formation, regulation, and evolution of Caenorhabditis elegans 3'UTRs. Nature 469: 97-101.

Katoh T, Sakaguchi Y, Miyauchi K, Suzuki T, Kashiwabara S, Baba T. 2009. Selective stabilization of mammalian microRNAs by $3^{\prime}$ adenylation mediated by the cytoplasmic poly(A) polymerase GLD-2. Genes Dev 23: 433-438.

Kim YK, Heo I, Kim VN. 2010. Modifications of small RNAs and their associated proteins. Cell 143: 703-709.

Lai EC. 2002. microRNAs are complementary to 3' UTR sequence motifs that mediate negative post-transcriptional regulation. Nat Genet 30: 363-364.

Landgraf P, Rusu M, Sheridan R, Sewer A, Iovino N, Aravin A, Pfeffer S, Rice A, Kamphorst AO, Landthaler M, et al. 2007. A mammalian microRNA expression atlas based on small RNA library sequencing. Cell 129: 1401-1414.

Lehrbach NJ, Armisen J, Lightfoot HL, Murfitt KJ, Bugaut A, Balasubramanian S, Miska EA. 2009. LIN-28 and the poly(U) polymerase PUP-2 regulate let-7 microRNA processing in Caenorhabditis elegans. Nat Struct Mol Biol 16: 1016-1020.

Lewis BP, Shih IH, Jones-Rhoades MW, Bartel DP, Burge CB. 2003. Prediction of mammalian microRNA targets. Cell 115: 787-798.

Li J, Yang Z, Yu B, Liu J, Chen X. 2005. Methylation protects miRNAs and siRNAs from a $3^{\prime}$-end uridylation activity in Arabidopsis. Curr Biol 15: 1501-1507.

Liu N, Abe M, Sabin LR, Hendriks G-J, Naqvi AS, Yu Z, Cherry S, Bonini NM. 2011. The exoribonuclease Nibbler controls 3' end processing of microRNAs in Drosophila. Curr Biol 21: 18881893.

Morin RD, O'Connor MD, Griffith M, Kuchenbauer F, Delaney A, Prabhu AL, Zhao Y, McDonald H, Zeng T, Hirst M, et al. 2008. Application of massively parallel sequencing to microRNA profiling and discovery in human embryonic stem cells. Genome Res 18: 610-621.

Newman MA, Thomson JM, Hammond SM. 2008. Lin-28 interaction with the Let-7 precursor loop mediates regulated microRNA processing. RNA 14: 1539-1549. 
Okamura K, Hagen JW, Duan H, Tyler DM, Lai EC. 2007. The mirtron pathway generates microRNA-class regulatory RNAs in Drosophila. Cell 130: 89-100.

Okamura K, Robine N, Liu Y, Liu Q, Lai EC. 2011. R2D2 organizes small regulatory RNA pathways in Drosophila. Mol Cell Biol 31: 884-896.

Park JE, Heo I, Tian Y, Simanshu DK, Chang H, Jee D, Patel DJ, Kim VN. 2011. Dicer recognizes the $5^{\prime}$ end of RNA for efficient and accurate processing. Nature 475: 201-205.

Persson H, Kvist A, Rego N, Staaf J, Vallon-Christersson J, Luts L, Loman N, Jonsson G, Naya H, Hoglund M, et al. 2011. Identification of new microRNAs in paired normal and tumor breast tissue suggests a dual role for the ERBB2/Her2 gene. Cancer Res 71: 78-86.

Ruby JG, Jan C, Player C, Axtell MJ, Lee W, Nusbaum C, Ge H, Bartel DP. 2006. Large-scale sequencing reveals 21U-RNAs and additional microRNAs and endogenous siRNAs in C. elegans. Cell 127: 1193-1207.

Ruby JG, Jan CH, Bartel DP. 2007a. Intronic microRNA precursors that bypass Drosha processing. Nature 448: 83-86.

Ruby JG, Stark A, Johnston WK, Kellis M, Bartel DP, Lai EC. 2007b. Evolution, biogenesis, expression, and target predictions of a substantially expanded set of Drosophila microRNAs. Genome Res 17: 1850-1864.

Rybak A, Fuchs H, Smirnova L, Brandt C, Pohl EE, Nitsch R, Wulczyn FG. 2008. A feedback loop comprising lin-28 and let-7 controls pre-let-7 maturation during neural stem-cell commitment. Nat Cell Biol 10: 987-993.

Seitz H, Ghildiyal M, Zamore PD. 2008. Argonaute loading improves the $5^{\prime}$ precision of both microRNAs and their miRNA strands in flies. Curr Biol 18: 147-151.
Shi W, Hendrix D, Levine M, Haley B. 2009. A distinct class of small RNAs arises from pre-miRNA-proximal regions in a simple chordate. Nat Struct Mol Biol 16: 183-189.

Siomi MC, Sato K, Pezic D, Aravin AA. 2011. PIWI-interacting small RNAs: The vanguard of genome defence. Nat Rev Mol Cell Biol 12: 246-258.

Spierings DC, McGoldrick D, Hamilton-Easton AM, Neale G, Murchison EP, Hannon GJ, Green DR, Withoff S. 2011. Ordered progression of stage-specific miRNA profiles in the mouse B2 B-cell lineage. Blood 117: 5340-5349.

Viswanathan SR, Daley GQ, Gregory RI. 2008. Selective blockade of microRNA processing by Lin28. Science 320: 97-100.

Witten D, Tibshirani R, Gu SG, Fire A, Lui WO. 2010. Ultra-high throughput sequencing-based small RNA discovery and discrete statistical biomarker analysis in a collection of cervical tumours and matched controls. BMC Biol 8: 58. doi: 10.1186/1741-7007-8-58.

Wu H, Neilson JR, Kumar P, Manocha M, Shankar P, Sharp PA, Manjunath N. 2007. miRNA profiling of naive, effector, and memory CD8 T cells. PLoS ONE 2: e1020. doi: 10.1371/journal. pone.0001020.

Yang JS, Lai EC. 2011. Alternative miRNA biogenesis pathways and the interpretation of core miRNA pathway mutants. Mol Cell 43: 892-903.

Yang JS, Maurin T, Robine N, Rasmussen KD, Jeffrey KL, Chandwani R, Papapetrou EP, Sadelain M, O'Carroll D, Lai EC. 2010. Conserved vertebrate mir-451 provides a platform for Dicerindependent, Ago2-mediated microRNA biogenesis. Proc Natl Acad Sci 107: 15163-15168.

Yu B, Yang Z, Li J, Minakhina S, Yang M, Padgett RW, Steward R, Chen X. 2005. Methylation as a crucial step in plant microRNA biogenesis. Science 307: 932-935. 

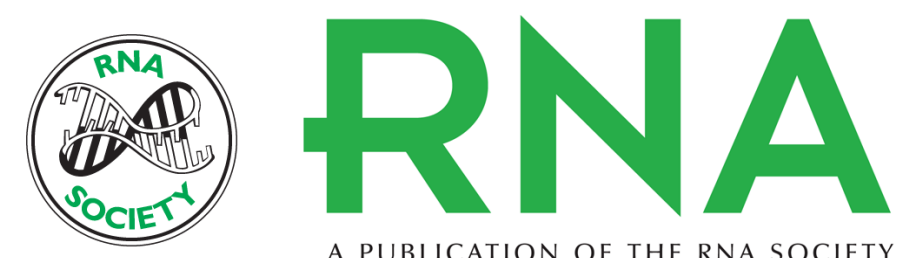

A PUBLICATION OF THE RNA SOCIETY

\section{Common and distinct patterns of terminal modifications to mirtrons and canonical microRNAs}

Jakub O. Westholm, Erik Ladewig, Katsutomo Okamura, et al.

RNA 2012 18: 177-192 originally published online December 21, 2011

Access the most recent version at doi:10.1261/rna.030627.111

Supplemental
Material http://rnajournal.cshlp.org/content/suppl/2011/12/12/rna.030627.111.DC1

References This article cites 51 articles, 21 of which can be accessed free at:

http://rnajournal.cshlp.org/content/18/2/177.full.html\#ref-list-1

License

Email Alerting Receive free email alerts when new articles cite this article - sign up in the box at the Service top right corner of the article or click here. 